\title{
Geodesic laminations with closed ends on surfaces and Morse index; Kupka-Smale metrics
}

Tobias H. Colding* and Nancy Hingston

\section{Introduction}

Let $M^{2}$ be a closed orientable surface with curvature $K$ and $\gamma \subset M$ a closed geodesic. The Morse index of $\gamma$ is the index of the critical point $\gamma$ for the length functional on the space of closed curves, i.e., the number of negative eigenvalues (counted with multiplicity) of the second derivative of length. Since the second derivative of length at $\gamma$ in the direction of a normal variation $u \boldsymbol{n}$ is $-\int_{\gamma} u L_{\gamma} u$ where $L_{\gamma} u=u^{\prime \prime}+K u$, the Morse index is the number of negative eigenvalues of $L_{\gamma}$. (By convention, an eigenfunction $\phi$ with eigenvalue $\lambda$ of $L_{\gamma}$ is a solution of $L_{\gamma} \phi+\lambda \phi=0$.) Note that if $\lambda=0$, then $\phi$ (or $\phi \boldsymbol{n}$ ) is a (normal) Jacobi field. $\gamma$ is stable if the index is zero. The index of a noncompact geodesic is the dimension of a maximal vector space of compactly supported variations for which the second derivative of length is negative definite. We also say that such a geodesic is stable if the index is 0 .

We give in this paper bounds for the Morse indices of a large class of simple geodesics on a surface with a generic metric. To our knowledge these bounds are the first that use only the generic hypothesis on the metric.

Theorem 1.1. For a generic metric on a closed surface, $M^{2}$, any geodesic lamination with closed ends has finitely many leaves and each leaf has finite Morse index.

Our second result is:

Theorem 1.2. For a generic metric on a closed surface, $M^{2}$, there is a bound for the Morse index of any collection of simple closed geodesics for which each limit is a geodesic lamination with closed ends.

A lamination on a surface $M^{2}$ is a collection $\mathcal{L}$ of smooth disjoint curves (called leaves) such that $\bigcup_{\ell \in \mathcal{L}} \ell$ is closed. Moreover, for each $x \in M$ there exists an open neighborhood $U$ of $x$ and a $C^{0}$ coordinate chart, $(U, \Phi)$, with $\Phi(U) \subset \mathbb{R}^{2}$ so that in these coordinates the leaves in $\mathcal{L}$ pass through $\Phi(U)$ in slices of the form

\footnotetext{
*The first author was partially supported by NSF Grants DMS 9803253 and DMS 0104453.
} 
$(\mathbb{R} \times\{t\}) \cap \Phi(U)$. A foliation is a lamination for which the union of the leaves is all of $M$ and a geodesic lamination is a lamination whose leaves are geodesics. The closure of the union of a collection of disjoint, simple, complete geodesics is always a geodesic lamination.

If $\ell \in \mathcal{L}$ is noncompact, then we set

$$
\ell_{+}=\bigcap_{s>0} \overline{\ell(s, \infty)}
$$

Since $\ell_{+}$is the intersection of nonempty nested closed sets it is closed and nonempty since $M$ is compact. Since $\bigcup_{\ell \in \mathscr{L}} \ell$ is closed, $\ell_{+} \subset \bigcup_{\ell \in \mathcal{L}} \ell$. Likewise we define $\ell_{-}$. A leaf $\ell \in \mathscr{L}$ is said to be isolated if for some $x \in \ell$ (hence all $x \in \ell$ ) there exists $\varepsilon=\varepsilon(x)>0$ such that $B_{\varepsilon}(x) \cap \tilde{\ell}=\emptyset$ for all $\tilde{\ell} \in \mathcal{L} \backslash\{\ell\}$. Note that $\ell_{-}, \ell_{+}$consist of nonisolated leaves. We say that a geodesic lamination $\mathcal{L}$ has closed ends if for each noncompact leaf $\ell \in \mathcal{L}$ both $\ell_{+}$and $\ell_{-}$are closed geodesics.

We will equip the space of metrics on a given manifold with the $C^{\infty}$-topology. A subset of the set of metrics on a given manifold is said to be residual if it is a countable intersection of open dense subsets. A statement is said to hold for a generic metric if it holds for all metrics in a residual set. The conclusions of Theorems 1.1 and 1.2 are true for a residual set of metrics that we call Kupka-Smale (KS-metrics). This hypothesis on the metric has a natural interpretation in both the dynamical systems and the variational contexts. Here are two versions of our hypothesis.

KS-metric (dynamical version):

(1) Every simple closed orbit of the geodesic flow whose Poincare map has real eigenvalues is hyperbolic.

(2) Every intersection of stable and unstable manifolds at a simple geodesic is transverse.

$K S$-metric (variational version): Let $\gamma$ be a simple geodesic.

(1) If $\gamma$ is periodic, there is no periodic Jacobi field without zeroes.

(2) If $\gamma$ is noncompact and has closed ends, there is no bounded Jacobi field without zeroes.

The above two conditions are equivalent under the additional condition that the metric is bumpy. A metric on a surface is bumpy if each closed geodesic is a nondegenerate critical point, i.e., $L_{\gamma} u=0$ implies $u \equiv 0$. Bumpy metrics are generic, [Ab], [An]. For convenience we will prove the conclusions of Theorems 1.1 and 1.2 for these "bumpy KS-metrics". For further discussion of these metrics, see Section 4 where we show that the set of bumpy KS metrics contain a residual set.

Here is the idea of the proof of Theorem 1.1: Unstable closed leaves in a fixed lamination are always isolated; thus we need to show that noncompact leaves are isolated and have finite index. In Theorem 1.1 part 1 of the KS-condition is applied 
to get a nice structure on the ends of noncompact leaves, and to ensure that these ends are isolated and countable. If an end leaf is closed and hyperbolic, this structure is striking: On each side of the end (limit) leaf there are two smooth circles of geodesics, each spiraling toward the limit leaf, one in each direction on each side; see Figure 1 and Corollary 3.4. Each circle foliates a tubular neighborhood of the given

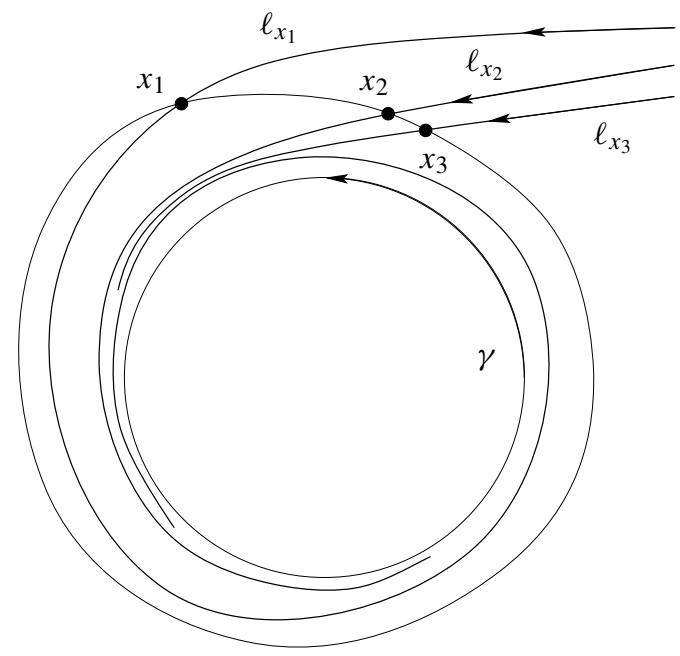

Figure 1. One of the four circles worth of noncompact geodesics spiraling into a simple closed strictly stable geodesic.

side of the end. These circles of noncompact geodesics are the stable and unstable manifolds of the end leaf, when viewed as a closed orbit of the geodesic flow on the unit tangent bundle of the surface. The second part of the KS condition ensures that, in a given lamination, leaves limiting on a given pair of ends are isolated, as the corresponding circles intersect transversely in a local (two-dimensional) section of the flow; see Figure 2. These noncompact leaves have finite index since index "stops accumulating" once they get close to the stable, hyperbolic, ends.

To prove Theorem 1.2 we first extract a converging subsequence of the given sequence of simple closed geodesics. The limit is easily seen to be a geodesic lamination with multiplicities; see the discussion preceding Proposition 3.10. On long stretches the geodesics in the subsequence will mimic the behavior of the limit lamination. By assumption this limit lamination has closed ends. The transversality of the intersection of the stable and unstable manifolds will then allow us to conclude finiteness of the indices for the converging subsequence of geodesics.

It is easy to see that the first part of Theorem 1.1 is false without the word "generic". One can construct a surface of revolution that has a geodesic lamination with infinitely many leaves. In this example all the leaves are stable and have closed ends. However 


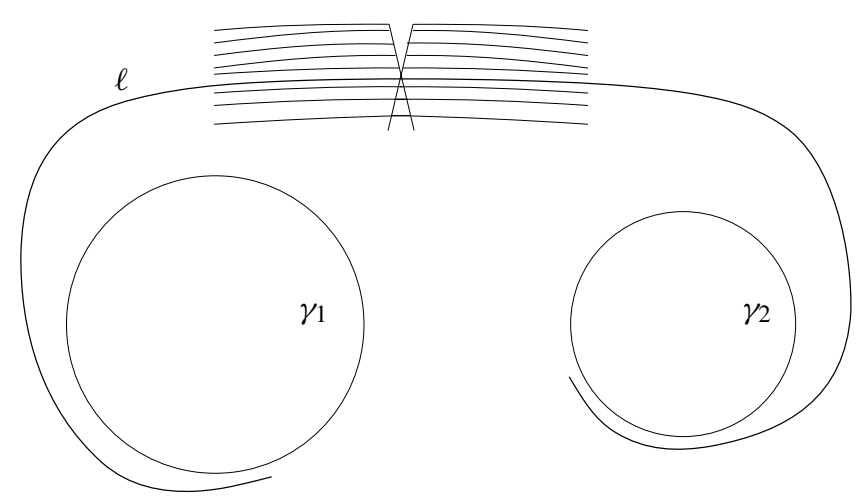

Figure 2. Transverse intersection of two circles worth of noncompact geodesics spiraling into two different simple closed strictly stable geodesics.

in [CH1] we showed that on any $M^{2}$, there exists a metric with a geodesic lamination with closed ends and infinitely many unstable leaves. Moreover, there exists such a metric which has no bound for the index of all simple closed geodesics.

General geodesic laminations on surfaces need not have closed ends; consider for instance a flat square torus with the foliation consisting of lines with a common irrational slope. In fact, on any surface there are (bumpy) metrics and geodesic laminations without closed ends:

Theorem 1.3. On any surface $M^{2}$, there exists an open (nonempty) set of metrics having geodesic laminations without closed ends. These laminations are limits of sequences of simple closed geodesics.

Our interest in whether the Morse index is bounded for simple closed geodesics on surfaces comes in part from its connection with the spherical space form problem; see [PiRu], [CM2] where Pitts and Rubinstein ask for such a bound for embedded minimal tori for a sufficiently large class of metrics on $\mathbb{S}^{3}$. Clearly obstructions to Morse index bounds for simple closed geodesics on surfaces give obstructions to Morse index bounds for embedded minimal tori on 3-manifolds (the most immediate generalization of simple closed geodesics on surfaces to 3-manifolds is embedded minimal tori with uniform curvature bounds). In addition new obstructions occur; [HaNoRu], [CD]. We believe that many of the ideas of this paper can be used to give bounds for the Morse indices of geodesics on surfaces and on embedded minimal tori (or more generally fixed genus) with uniform curvature bounds on 3-manifolds with generic metrics. In fact, the arguments given here should be useful even for surfaces without curvature bounds; for instance on closed 3-manifolds with positive scalar curvature any complete stable minimal surface is necessarily compact and in 
fact either topologically $\mathbb{S}^{2}$ or $\mathbb{R} \boldsymbol{P}^{2}$. Thus "ends" of embedded minimal annuli (even without curvature bounds) in such manifolds are closed; see [CD] for more discussion on this.

Throughout this paper $M^{2}$ is a closed orientable surface with a Riemannian metric, $\mathcal{L}$ is a geodesic lamination, and when $x \in M, r_{0}>0$, and $D \subset M$, then we let $B_{r_{0}}(x)$ denote the ball of radius $r_{0}$ centered at $x$ and $T_{r_{0}}(D)$ the $r_{0}$-tubular neighborhood of $D$. Moreover, if $x, y \in M$, then $\gamma_{x, y}:\left[0, \operatorname{dist}_{M}(x, y)\right] \rightarrow M$ will denote a minimal geodesic from $x$ to $y$. Whenever we look at a single geodesic it will always be assumed to be parameterized by arclength.

We are grateful to Camillo De Lellis for making the illustrations.

\section{Geodesic laminations on surfaces}

We will often implicitly use the following simple fact: If $\gamma \subset M^{2}$ is a simple closed geodesic, then there exists $\delta=\delta(\gamma)>0$ such that the nearest point projection $\Pi_{\gamma}: T_{\delta}(\gamma) \rightarrow \gamma$ is well defined. Moreover, if $\tilde{\gamma}:[0,1] \rightarrow T_{\delta}(\gamma)$ is a geodesic, then

$$
\left.|| d \Pi_{\gamma}\right|_{\tilde{\gamma}}|-1|<\psi(\delta)
$$

where $\lim _{\delta \rightarrow 0} \psi(\delta)=0$. Note that this just says that the geodesics $\gamma$ and $\tilde{\gamma}$ are nearly parallel. If $\gamma$ is oriented, then we say that $\tilde{\gamma}:[0,1] \rightarrow T_{\delta}(\gamma)$ has the same orientation as $\gamma$ if $\left|d \Pi_{\gamma} \tilde{\gamma}^{\prime}-\gamma^{\prime}\right|<\psi(\delta)$. We will most often assume that this is the case.

We will assume in what follows some knowledge of Jacobi fields and indices of geodesics; see e.g. [Kl] or [Sp]. Three facts will be particularly important:

1) A Jacobi field $J$ is uniquely determined by the values $\left(J(t), J^{\prime}(t)\right)$ for any $t$.

2) A complete geodesic (closed or noncompact) is stable if and only if it has no Jacobi field with more than one zero. (In the closed case we of course mean Jacobi field with the same periodicity as the geodesic.)

3) A noncompact geodesic has finite index if and only if there is a bound for the number of zeros of any nontrivial Jacobi field along it.

To show that certain geodesics are stable (or have bounded Morse index) it is sometimes useful to apply the following standard fact: A Schrödinger operator $L u=$ $u^{\prime \prime}+K u$ is nonpositive $(-L \geq 0)$ if it has a positive supersolution $\phi$ (that is $\phi>0$ and $L \phi \leq 0)$. This follows since $-(\log \phi)^{\prime \prime} \geq K+\left|(\log \phi)^{\prime}\right|^{2}$ and hence if $f$ is a compactly supported function, then integration by parts and the Cauchy-Schwarz 
inequality yields

$$
\begin{aligned}
\int f^{2} K+\int f^{2}\left|(\log \phi)^{\prime}\right|^{2} & \leq-\int f^{2}(\log \phi)^{\prime \prime} \\
& =2 \int f f^{\prime}(\log \phi)^{\prime} \leq \int f^{2}\left|(\log \phi)^{\prime}\right|^{2}+\int\left(f^{\prime}\right)^{2} .
\end{aligned}
$$

Thus, $-\int f L f=-\int f\left(f^{\prime \prime}+K f\right) \geq 0$.

Lemma 2.1. Let $\gamma$ be a strictly stable $\left(-L_{\gamma}>0\right)$ simple closed geodesic on $M^{2}$. There exists $\delta=\delta(\gamma)>0$, such that any geodesic segment contained in $T_{\delta}(\gamma)$ with length $\geq 1$ is stable.

Proof. Since $\gamma$ is strictly stable, then $-L_{\gamma}>0$ so if we let $\lambda_{1}$ be the first eigenvalue and $\phi$ a corresponding eigenfunction, then $\lambda_{1}>0$ and $\phi^{2}>0$. In particular $-L_{\gamma}|\phi|=\lambda_{1}|\phi|>0$. Let $\tilde{\gamma} \subset T_{\delta}(\gamma)$ be a geodesic segment with length $\geq 1$ and set $\tilde{\phi}=\phi \circ \Pi_{\gamma}$, then $|\tilde{\phi}|>0$ and (by (2.1)) $-L_{\tilde{\gamma}}|\tilde{\phi}|>0$. The lemma now follows from the remarks preceding it.

Let $\gamma$ be a closed geodesic with universal cover $\tilde{\gamma}$. Then

1) $\gamma$ is stable if and only if $\tilde{\gamma}$ has no Jacobi field $J: \mathbb{R} \rightarrow \mathbb{R}$ with 2 zeroes.

2) $\gamma$ is strictly stable if and only if $\gamma$ is stable and $\tilde{\gamma}$ has no periodic Jacobi field.

The "only if" part of each statement follows by contradiction from the simple argument of Lemma 2.1 when applied to a first eigenfunction $\phi$, with $f$ the restriction of $J$ for 1) to an interval between two zeroes, and for 2) to one period of $J$. The "if" part of 2) is clear; to see the "if" part of 1) we argue using (2.2): Let $\chi_{n}$ be the cutoff function (we may assume that Length $(\gamma)=1$ ),

$$
\chi_{n}(t)= \begin{cases}1 & \text { for }|t| \leq n^{2} \\ 1-\left(|t|-n^{2}\right) / n & \text { for } n^{2}<|t| \leq n^{2}+n \\ 0 & \text { otherwise }\end{cases}
$$

If $f$ is a function on $\gamma, \tilde{f}$ its lift to $\tilde{\gamma}$, set $f_{n}=\tilde{f} \chi_{n}$, and $\phi_{n}=J_{n}$ (where $J_{n}$ is a Jacobi field with $J_{n}\left(-n^{2}-n\right)=0$ and $J_{n} \mid\left(-n^{2}-n, \infty\right)>0$. Then by (2.2) for $f_{n}$

$$
\begin{aligned}
2 n^{2} \int_{\gamma} K f^{2} & \leq \int K \tilde{f}^{2} \chi_{n}^{2} \leq \int\left[\left(\tilde{f} \chi_{n}\right)^{\prime}\right]^{2} \\
& \leq \int\left[(\tilde{f})^{\prime}\right]^{2} \chi_{n}^{2}+\frac{1}{2 n} \int_{n^{2}<|t| \leq n^{2}+n}\left[(\tilde{f})^{2}+\left(\tilde{f}^{\prime}\right)^{2}\right]+\int(\tilde{f})^{2}\left(\chi_{n}^{\prime}\right)^{2} \\
& \leq 2 n(n+2) \int_{\gamma}\left(f^{\prime}\right)^{2}+3 \int_{\gamma}(f)^{2}
\end{aligned}
$$


Dividing (2.4) through by $2 n^{2}$ and letting $n \rightarrow \infty$ gives $-\int_{\gamma} f L f=-\int_{\gamma} f\left(f^{\prime \prime}+\right.$ $K f) \geq 0$.

We will also use the following two well-known facts; see e.g. [Kl] or [Sp]. One can also see 4) below by arguing as in 1) and 2) above:

3) A Jacobi field $J$ is uniquely determined by the values $\left(J(t), J^{\prime}(t)\right)$ for any $t$.

4) A noncompact geodesic is stable if and only if it has no Jacobi field with more than one zero and it has finite index if and only if there is a bound for the number of zeros of any nontrivial Jacobi field along it.

Note that 1) and 4) together imply that $\gamma$ is stable if and only if $\tilde{\gamma}$ is.

Lemma 2.2. Any nonisolated leaf $\ell$ of $\mathcal{L}$ is stable.

Proof. If $\ell$ had a Jacobi field with 2 zeroes, then so would every sufficiently nearby (in the unit tangent bundle) geodesic. But between $\ell$ and any nearby geodesic $\ell_{i}$ which does not intersect $\ell$, we can find (using $\ell$ and $\ell_{i}$ as barriers) a stable geodesic $\eta$ which has no Jacobi field with 2 zeroes.

From the definition of a lamination and Lemma 2.2 one easily shows:

Lemma 2.3. Each $\ell_{+}$is connected (as a subset of $M$ ). Moreover, given $x \in \ell_{+}$, then $x \in \ell_{0} \subset \ell_{+}$for some $\ell_{0} \in \mathcal{L} ; \ell_{0}$ is said to be a limit leaf and is stable.

We will need the following result.

Lemma 2.4. If $\ell \in \mathcal{L}$ is noncompact and $\ell_{+}$contains a closed geodesic $\sigma$, then $\ell_{+}=\sigma$ and $\ell$ spirals monotonically toward $\ell_{+}$.

Proof. Since $\ell$ is simple and complete and does not intersect $\sigma$, once $\ell$ gets into a small tubular neighborhood of $\sigma$, then one of the two "directions" of $\ell$ must be completely contained in a small tubular neighborhood of $\sigma$. Now using that $\sigma \subset \ell_{+}$ it follows from this that the "forward direction" of $\ell$ is actually contained in a small tubular neighborhood of $\sigma$ and hence (again since $\sigma \subset \ell_{+}$) must spiral toward $\sigma$ monotonically.

We say that a geodesic $\gamma_{2}:\left[0, k_{2}\right] \rightarrow M^{2}$ can be written as a normal graph over a geodesic $\gamma_{1}:\left[0, k_{1}\right] \rightarrow M$ by a function $u$ (on $\left[0, k_{1}\right]$ ) if there is a diffeomorphism $\alpha:\left[0, k_{1}\right] \rightarrow\left[0, k_{2}\right]$ such that for all $t \in\left[0, k_{1}\right]$,

$$
\gamma_{2}(\alpha(t))=\exp _{\gamma_{1}(t)}\left(u(t) \boldsymbol{n}_{\gamma_{1}}(t)\right) .
$$

The next corollary follows from Lemma 2.4. 
Corollary 2.5. Suppose that $\mathcal{L}$ has closed ends. If $\ell_{i}, \ell \in \mathcal{L}$ and $\ell$ is noncompact with $\ell_{i}(0) \rightarrow \ell(0)$, then, for $i$ sufficiently large, $\ell_{i_{ \pm}}=\ell_{ \pm}$and $\ell_{i}$ is a normal graph over $\ell$.

Recall that we equip the space of $C^{\infty}$ metrics on a closed surface $M^{2}$ with the $C^{\infty}$-topology and we write $g_{i} \rightarrow g$ if $\left|g-g_{i}\right|_{C^{k}} \rightarrow 0$ for all $k$. Most of the next lemma will be needed only in Section 4 .

Lemma 2.6 (Lemma B.1 of [CH1]). Suppose that the metric $g$ on $M$ is bumpy. For each $L>0$, there exists at most finitely many closed geodesics of length $<L$. Moreover, if $L$ is not equal to the length of any closed geodesic in $g$, then in a neighborhood of $g$ each metric has precisely as many (simple) closed stable geodesics of length $<L$ as $g$. Finally, if $g_{i} \rightarrow g$ and $\left\{\gamma_{i, k}\right\},\left\{\gamma_{k}\right\}$ are the (simple) closed stable geodesics in $g_{i}, g$, respectively, of length $<L$, then $\gamma_{i, k} \rightarrow \gamma_{k}$ for $i \rightarrow \infty$ and each $k$.

In the remainder of this section the metric on $M^{2}$ is bumpy and $\mathcal{L}$ has closed ends. For a bumpy metric (or for a metric with either version of the condition KS (1)), each element in a collection of disjoint simple closed geodesics is isolated. Thus $\mathcal{L}$ contains finitely many closed leaves $\eta_{1}, \ldots, \eta_{m}$; these are the only limit leaves. Choose $\varepsilon>0$ so that:

$$
\operatorname{dist}\left(\eta_{j}, \eta_{k}\right)>2 \varepsilon \quad \text { for } j \neq k
$$

$T_{\mathcal{E}}\left(\eta_{j}\right) \cap \ell$ is graphical over $\eta_{j}$ for $1 \leq j \leq m$ and $\ell \in \mathcal{L}$; and $\Pi: T_{\mathcal{E}}\left(\bigcup \eta_{j}\right) \rightarrow \bigcup \eta_{j}$ is smooth. Using the local product structure, there exist $C>0$ and $S$ with $\partial S$ smooth so that

$$
\bigcup T_{\varepsilon / C}\left(\eta_{j}\right) \subset S \subset \bigcup T_{\varepsilon}\left(\eta_{j}\right)
$$

and $\partial S$ intersects $\mathcal{L}$ transversely. $S_{j}$ denotes the component of $S$ containing $\eta_{j}$.

Corollary 2.7. Suppose that the metric on $M$ is bumpy and $\mathcal{L}$ has closed ends. Let $\eta_{j}$ and $S$ be as above. There exists $\rho<\infty$ so that, for each $\ell \in \mathcal{L}$, each component $\alpha$ of $\ell \backslash S$ has Length $(\alpha) \leq \rho$.

Proof. This follows by compactness.

Using the local product structure, Corollary 2.7 implies that there are collections $T_{j}, 1 \leq j \leq \bar{m}$, of components $\alpha$ of $\bigcup_{\mathcal{L}} \ell \backslash S$ so that each $\alpha \in T_{j}$ spirals between $\eta_{1_{j}}$ and $\eta_{2_{j}}$. Hence,

$$
\min _{j_{1} \neq j_{2}} \operatorname{dist}\left(\bigcup_{\alpha \in T_{j_{1}}} \alpha, \bigcup_{\alpha \in T_{j_{2}}} \alpha\right)=\bar{\varepsilon}>0 .
$$




\section{Morse index bounds for bumpy Kupka-Smale metrics}

Let $M^{2}$ be a closed surface with a bumpy metric. In this section we discuss the stability of the leaves of a geodesic lamination. The first goal is to prove Corollary 3.7, which says that a nonisolated leaf in a geodesic lamination with closed ends implies a non-transverse intersection of two circles which will be described below. From this corollary we will then be able to give a condition (KS-metrics) on a metric on $M$ which will imply that all geodesic laminations with closed ends have finitely many leaves, and that there is a bound for the Morse index of simple closed geodesics; see Propositions 3.9 and 3.10. In Section 4 we will see that this condition is generically satisfied.

If $\gamma:\left[t_{1}, t_{2}\right] \rightarrow M^{2}$ is a geodesic, then we let $P_{t_{2}, t_{1}}$ denote the (relative) linear Poincaré map which describes to first order how nearby geodesics advance along $\gamma$. That is, if $(a, b) \in \mathbb{R}^{2}$, then $P_{t_{2}, t_{1}}(a, b)=\left(J\left(t_{2}\right), J^{\prime}\left(t_{2}\right)\right)$ where $J$ is the Jacobi field on $\gamma$ with $J\left(t_{1}\right)=a$ and $J^{\prime}\left(t_{1}\right)=b$. Note that $P_{t_{2}, t_{1}}=P_{t_{2}, t} P_{t, t_{1}}$. Set

$$
R_{\gamma}(t)=\left(\begin{array}{cc}
0 & 1 \\
-K(\gamma(t)) & 0
\end{array}\right)
$$

By the Jacobi equation $\frac{d}{d t} P_{t, t_{1}}=R_{\gamma}(t) P_{t, t_{1}}$; since $\operatorname{Tr}\left(R_{\gamma}\right)=0$ and $P_{t_{1}, t_{1}}$ is the identity it follows that $P_{t, t_{1}} \in \mathrm{SL}(2, \mathbb{R})$. Observe that if $\gamma:\left[0, s_{\gamma}\right] \rightarrow M$ is closed, then $P_{\gamma}=P_{s_{\gamma}, 0}$ is the usual linear Poincaré map.

The existence of a Jacobi field along $\gamma$ with zeroes at $t_{1}$ and $t_{2}$ is equivalent to the fact that $P_{t_{2}, t_{1}}$ (as a linear map from $\mathbb{R}^{2}$ to itself) takes the $y$-axis to itself; thus we will want to keep an eye on the $y$-axis as $P_{t, t_{1}}$ acts on $\mathbb{R}^{2}$. Note that the 1 in the upper right corner of $R_{\gamma}$ means that if we watch the motion of a vector $(a, b)$ under $P_{t, t_{1}}$, then at a time $t_{2}$ when the vector hits the $y$-axis (i.e. when $\left.P_{t_{2}, t_{1}}(a, b)=(0, y)\right)$, the vector is moving clockwise, i.e. $\frac{d}{d t} P_{t, t_{1}}(a, b)=(y, 0)$. (This agrees with common sense: If $J\left(t_{2}\right)=0$ and $J^{\prime}\left(t_{2}\right)=y$, then $J(t)$ has the same sign as $y$ for $t>t_{2}$.)

If the metric on $M$ is bumpy, and $\gamma$ is a closed limit leaf of a geodesic lamination, then $\gamma$

1) is simple,

2) has no Jacobi field $J: \mathbb{R} \rightarrow \mathbb{R}$ with 2 zeroes,

3) has no periodic Jacobi field.

If $\gamma$ is closed, it is clear by the above discussion and continuity that if $P_{\gamma}=P_{s_{\gamma}, 0}$ does not have a positive real eigenvalue (i.e. if $P_{\gamma}$ does not fix the direction of some vector in $\mathbb{R}^{2}$ ), then the path $P_{t, 0}, 0 \leq t \leq s_{\gamma}$, rotates each vector in $\mathbb{R}^{2}$ clockwise by a positive amount. However, since $P_{m s_{\gamma}+t, 0}=P_{t, 0} P_{s_{\gamma}, 0}^{m}$, in that case eventually the $y$-axis will be mapped to itself, causing a Jacobi field with 2 zeroes. Thus if $\gamma$ is simple closed and strictly stable, the eigenvalues of $P_{\gamma}$ are of the form $\lambda$ and $1 / \lambda$ 
where $0<\lambda<1$; it follows that $P_{\gamma}$ has a basis (not necessarily orthogonal) of eigenvectors. (Note that 1 cannot be an eigenvalue by 3 ).)

A local section $\Sigma$ of the geodesic flow along $\gamma$ is obtained as follows; [Bi], [MS]. Pick $t_{0}$, and construct a geodesic $\tau$ on $M$ transverse to $\gamma^{\prime}\left(t_{0}\right)$ at $\gamma\left(t_{0}\right)$. Let $\Pi$ be the projection from the unit tangent bundle $T_{1} M$ onto $M$. The surface $\Sigma \subset T_{1} M$ is the intersection of a neighborhood of $\gamma^{\prime}\left(t_{0}\right)$ with the set $\Pi^{-1}(\tau)$. Each point in $\Sigma$ corresponds (by giving an initial tangent vector) to a geodesic near $\gamma$. If $\gamma$ is closed, by following the geodesics around we obtain the $\left(C^{1}\right)$ Poincaré map $\mathcal{P}: \Sigma \rightarrow \Sigma$ with fixed point $\gamma^{\prime}(0)$. (Strictly speaking we will need to make the domain of $\mathcal{P}$ smaller in order to get the range inside $\Sigma$.) The derivative of $\mathcal{P}$ at $\gamma^{\prime}(0)$ is the linear Poincaré map $P=P_{\gamma}$.

We will use without proof the following lemma, which says that an appropriate limit of geodesics is a Jacobi field. We decline to put a topology on the set of geodesics on $M$. However, very loosely speaking, if we think of the space of Jacobi fields along $\gamma$ as the tangent space to the set of geodesics at $\gamma$, then the lemma says that a neighborhood of $\gamma$ in the set of geodesics is diffeomorphic to a neighborhood of $\gamma^{\prime}\left(t_{0}\right)$ in $\Sigma$. The "diffeomorphism" takes a geodesic $\sigma$ to its tangent vector $\sigma^{\prime}(t)$ at the time $t$ when it crosses $\tau$, and a Jacobi field along the geodesic to the values $\left(J(t), J^{\prime}(t)\right)$ at that time.

Lemma 3.1. Let $\gamma$ be a geodesic, and for $i \geq 1$ let $u_{i}(t)$ be the normal graph over $\gamma$ of a geodesic $\gamma_{i}$. Assume that $\lim _{i \rightarrow \infty}\left\|\left(u_{i}(0), u_{i}^{\prime}(0)\right)\right\|=0$, and that the limit

$$
\lim _{i \rightarrow \infty}\left(u_{i}(0), u_{i}^{\prime}(0)\right) /\left\|\left(u_{i}(0), u_{i}^{\prime}(0)\right)\right\|
$$

exists. Fix $t_{0}$. Then $\lim _{i \rightarrow \infty} u_{i} / \|\left(u_{i}\left(t_{0}\right), u_{i}^{\prime}\left(t_{0}\right) \|\right.$ exists and represents a Jacobi field $J$ with

$$
\left(J(t), J^{\prime}(t)\right)=\lim _{i \rightarrow \infty}\left(u_{i}(t), u_{i}^{\prime}(t)\right) /\left\|\left(u_{i}\left(t_{0}\right), u_{i}^{\prime}\left(t_{0}\right)\right)\right\|
$$

for all $t$. Conversely, any Jacobi field is the limit of a 1-parameter family of geodesics (though it may be that none of these geodesics is the normal graph of a function $u(t)$ defined for all $t$ ).

Suppose now that $\gamma$ is simple closed and strictly stable. The lemma that follows says that the Poincare map $\mathcal{P}$ has the same behavior as its derivative $P$ : It has one contracting direction (eigenspace for $\lambda$ ) and one expanding direction (eigenspace for $1 / \lambda)$.

Lemma 3.2 (See [HiPu]). Let $\gamma$ be simple closed and strictly stable. If $\Sigma$ is sufficiently small, then there is a $C^{1}$ curve $Y$ through the origin in $\Sigma$ with the property that, for all $x \in \Sigma$,

$$
\lim _{n \rightarrow \infty} \mathcal{P}^{n} x=0 \Longleftrightarrow x \in Y \Longleftrightarrow \mathcal{P}^{n} x \in \Sigma \text { for all } n>0 .
$$


Thus a geodesic $\tau$ near $\gamma$ has $\tau_{+}=\gamma$ if and only if the point $x$ in $\Sigma$ corresponding to $\tau$ lies on $Y$. $Y$ is called the stable manifold of $\mathcal{P}$.

Corollary 3.3. Let $\gamma$ be simple closed and strictly stable. If $\ell$ is a noncompact simple geodesic with $\ell_{+}=\gamma($ or $\ell=\gamma)$, then $\ell$ has a unique Jacobi field $J_{+}$with the normalization $X_{+}(0) \in\{(\cos \theta, \sin \theta) \mid-\pi / 2<\theta \leq \pi / 2\}$, where $X_{+}(t)=$ $\left(J_{+}(t), J_{+}^{\prime}(t)\right)$, and with $\left\|J_{+}(t)\right\|$ bounded for $t>0$. For this vector field there exists $C$ and $\alpha>0$ such that for $t>0$

$$
\left\|X_{+}(t)\right\| \leq C \exp (-\alpha t) .
$$

Proof. Let $x$ be a point in $\Sigma$ representing $\ell$; this means $x=\ell^{\prime}\left(t_{0}\right)$. A vector in the tangent space to $\Sigma$ at $x$ represents (by giving the initial values $J\left(t_{0}\right)$ and $J^{\prime}\left(t_{0}\right)$ ) a Jacobi field along $\ell$. By Lemma 3.1, the derivative $d_{x} \mathcal{P}$ describes how the Jacobi field $J$ advances along one loop of $\ell$. Using (3.2) it is clear that a tangent vector to the stable manifold $X$ at $x$ represents a Jacobi field with the desired property.

Corollary 3.4 (See Figure 1). Let $\gamma$ be simple closed and strictly stable. Then there are four "circles" of noncompact geodesics limiting on $\gamma$. That is, on each side of $\gamma$ in $M$, and for each orientation of $\gamma$ there is a $C^{1}$ map $\mathbb{S}^{1} \rightarrow T_{1} M$ which gives a bijection between the circle $\mathbb{S}^{1}$ and the set of geodesics $\ell$ with $\ell_{+}=\gamma$ which limit on $\gamma$ from the given side of $M$ with the given orientation.

Proof. Implicit in the statement of the corollary is the map from $T_{1} M$ to the set (untopologized) of geodesics on $M$. One way to parameterize the circle, in the set of geodesics, is to use the segment of the stable manifold $Y$ between two consecutive points $x$ representing a single geodesic $\ell$ with $\ell_{+}=\gamma$. In order to lift the circle to a $C^{1}$ map $\mathbb{S}^{1} \rightarrow T_{1} M$, it is clear how to reparameterize along the segment in order to get the ends to match up. Once this is done, the image of $\mathbb{S}^{1}$ in $T_{1} M$ will be a circle close to the curve of tangents of $\gamma$, whose image in $M$ lies on the given side of $\gamma$.

If a geodesic $\ell$ lies in one of these four circles of geodesics given by Corollary 3.4, then the vector field $J_{+}$along $\ell$ can be thought of as a tangent vector to the circle.

Corollary 3.5. Let $\gamma$ be simple closed and strictly stable. There exists a neighborhood $T$ of $\gamma$ such that for all $x \in T \backslash\{\gamma\}$ there is a unique (maximal) geodesic $\ell_{x}:(a, \infty) \rightarrow T$ with the same orientation as $\gamma$ and $x \in \ell_{x}$. Moreover, $a>-\infty$, $\partial \ell_{x} \in \partial T, \ell_{x} \subset T \backslash\{\gamma\}, \ell_{x}$ is simple, $\left(\ell_{x}\right)^{+}=\gamma$, and $\mathcal{F}=\left\{\ell_{x}\right\}_{x \in \partial T} \cup\{\gamma\}$ is a geodesic foliation of $T$.

Proof. Let $T$ have as its boundary the image in $M$ of the circle in $T_{1} M$ given in the previous corollary. (This will need to be done once on each side of $\gamma$.) To get the 
foliation structure, and the simplicity of $\ell_{x}$, we use the fact that the derivative of the composite

$$
Y \longrightarrow \Sigma \underset{\Pi}{\longrightarrow} M
$$

at the point $\gamma^{\prime}\left(t_{0}\right)$ is nontrivial. The latter fact can be seen as follows: A tangent vector to $Y$ at $\gamma^{\prime}\left(t_{0}\right)$ gives the initial values $\left(J\left(t_{0}\right), J^{\prime}\left(t_{0}\right)\right)$ of a Jacobi field which returns after one loop around $\gamma$ as a multiple $1 / \lambda$ of itself. Since $\gamma$ is stable and thus has no Jacobi field with two zeroes, $J\left(t_{0}\right) \neq 0$ and thus the image under $\Pi$ is nonzero.

There is a direct way (using the appendix of [CH1]) of getting Corollaries 3.4, 3.5 without appealing to Lemma 3.2. Namely, by appendix A of [CH1] there exists a strictly convex function $F$ defined in a neighborhood $\{F \leq \varepsilon\}$ (where $\varepsilon>0$ is sufficiently small) of $\gamma$. (In Figure 1 the curve circling $\gamma$ is meant to represent a level set of $F$.) Note that each side of $\gamma$ in this neighborhood is convex and homeomorphic to a cylinder. A straightforward convergence argument shows that for each $x \in\{F=\varepsilon\}$ there exists a simple stable geodesic $\ell_{x} \subset\{F \leq \varepsilon\}$ with $x \in \ell_{x}$ and $\left(\ell_{x}\right)_{+}=\gamma$ as in Corollary 3.5. That $\ell_{x}, \ell_{y}$ do not cross (and that $\bigcup_{x} \ell_{x}=\{F \leq \varepsilon\} \backslash\{\gamma\}$ ) follows easily from Lemma 3.1 using the linear Poincaré map. Note that in this case each orientation of each component of $\{F=\varepsilon\}$ gives a parameterization of one of the four circles.

Corollary 3.6. Let $\gamma$ be simple closed and strictly stable. There exists $\varepsilon>0$ such that if $X_{-}$is the vector field defined on $T_{\varepsilon}(\gamma) \backslash\{\gamma\}$ by $X_{-}(x)=\tilde{\gamma}^{\prime}(0)$ where $\tilde{\gamma}$ is a noncompact geodesic with $\tilde{\gamma}_{-}=\gamma$ and $\tilde{\gamma}(0)=x$, then $X_{-}$is $C^{1}$. $X_{-}$and its (first) derivatives are also continuous functions of the metric on $M$. Moreover, there exists a $C^{2}$ curve c orthogonal to $X_{-}$and such that $\partial c \in \partial T_{\varepsilon}(\gamma) \cup \gamma$.

The statement that $X_{-}$is continuous in the metric makes sense in light of Lemma 2.6. The vector field $X_{-}$is also locally defined and $C^{1}$ with respect to the metric in a neighborhood of a point $\ell\left(t_{0}\right)$ if $\ell_{-}=\gamma, \gamma$ is simple closed and strictly stable, and if the vector field $J_{-}$along $\ell$ has $J_{-}\left(t_{0}\right) \neq 0$.

Proof of Corollary 3.6. The continuous dependence of the derivatives of $X_{-}$upon the metric follows from (in order) the continuity of the geodesic flow in the metric; the fact that the Poincaré map $\mathcal{P}$ is $C^{1}$, with derivatives depending continuously upon the metric; the fact that the stable manifold $Y$ is $C^{1}$, with derivatives depending continuously upon the metric. It can also be seen more directly using only the continuity of the geodesic flow and general dynamic properties of the flow near $\gamma$.

The next corollary is central to what follows. A noncompact leaf $\ell$ in a geodesic lamination with $\ell_{+}, \ell_{-}$simple closed and strictly stable geodesics lies in the intersection of two circles of geodesics, corresponding to its limit leaves $\ell_{+}$and $\ell_{-}$. If $\ell$ is not 
an isolated leaf, say $\ell_{i} \in \mathcal{L}$ with $\ell_{i}(0) \rightarrow \ell(0)$, then as in Corollary 2.5 the leaves $\ell_{i}$ have the same limit leaves and thus also lie in the intersection of the same two circles of geodesics. Corollary 3.7 can be thought of as saying that under these assumptions, the two circles of geodesics have a common tangent vector at the point $\ell$; thus the two circles are intersecting non-transversely. The vector field $J_{+}$(respectively $J_{-}$) defined in Corollary 3.3 should be thought of as the tangent vector to the circle of geodesics ending at $\ell_{+}$(respectively $\ell_{-}$) at the point $\ell$.

Corollary 3.7. Let $\mathcal{L}$ be a geodesic lamination on $M$. If $\ell, \ell_{i} \in \mathcal{L}$ are (distinct) noncompact, $\ell_{+}, \ell_{-}$are strictly stable simple closed geodesics, and $\ell_{i}(0) \rightarrow \ell(0)$, then $\ell$ is stable and there exists a bounded (nontrivial) Jacobi field on $\ell$. Thus $J_{+}=J_{-}$.

Proof. As in Corollary 2.5 we can assume that $\ell_{i_{ \pm}}=\ell_{ \pm}$, and that $\ell_{i}$ is the normal graph of a function $u_{i}(t)$ over $\ell$. Let $\Sigma$ be a local section near the point $\ell_{+}^{\prime}(0)$. By Lemma 3.2, $\ell$ and $\ell_{i}$ all correspond to points in $\Sigma$ lying on the stable manifold $Y$. By Lemma 3.1, $\left.\lim _{i \rightarrow \infty} u_{i} / \|\left(u_{i}(0)\right), u_{i}^{\prime}(0)\right) \|$ exists and is equal to $J_{+}$. By the same reasoning, $\lim _{i \rightarrow \infty} u_{i} /\left\|\left(u_{i}(0), u_{i}^{\prime}(0)\right)\right\|=J_{-}$.

Corollary 3.8. For a closed surface with a bumpy metric, the dynamical and variational versions of the Kupka-Smale hypothesis are equivalent. A bumpy metric is $K S$ (by either definition) if and only if for each simple stable (noncompact) geodesic with closed ends, $J_{+} \neq J_{-}$.

Proposition 3.9. Let $M^{2}$ be a closed surface with a bumpy metric and let $\mathcal{L}$ be a geodesic lamination with closed ends. Then each leaf has finite index and $\mathcal{L}$ has at most finitely many closed leaves each of which is either isolated or strictly stable. Moreover, if $\mathcal{L}$ has infinitely many leaves, then there exists a stable noncompact leaf with a (nontrivial) bounded Jacobi field.

Proof. This follows by combining Lemmas 2.2, 2.1, and 2.4 with Corollary 3.7. The index of each noncompact leaf is finite since by Lemma 2.1 and Lemma 2.4 index only accumulates on a finite interval away from the two ends.

There are two different ways of proving our bounds on the Morse indices. One is to use exclusively the Poincaré map and Jacobi fields (this is the way we will prove Proposition 3.10 below). The other is to construct positive supersolutions of the Jacobi equation. A particularly simple example of the second is given in Lemma 2.1. Each approach uses the eigenvalue gap, that is that there are no bounded (nontrivial) Jacobi fields on simple noncompact geodesics with closed ends.

Before proving our next result we will need a brief discussion on convergence of a sequence of simple closed geodesics $\left\{\gamma_{i}\right\}$ in a closed orientable surface $M^{2}$. Let 
$r_{0}>0$ be sufficiently small depending only on $\max _{M}|K|$ and the injectivity radius of $M$. Fix $x \in M$, then $B_{r_{0}}(x) \cap \gamma_{i}$ is the union of disjoint geodesics segments of length at most $2 r_{0}$ for each $i$. Note that any two such that come close to each other are "almost parallel". In fact it follows easily from the equation for geodesics that for each $i$ there is a coordinate chart $B_{r_{0}}(x) \rightarrow B_{r_{0}}(0) \subset \mathbb{R}^{2}$ such that each component of $B_{r_{0}}(x) \cap \gamma_{i}$ is mapped to a line segment of the form $B_{r_{0}}(0) \cap(\mathbb{R} \times\{t\}) \subset \mathbb{R}^{2}$. In this way one can think of each $\gamma_{i}$ as a geodesic lamination where the size of the coordinate chart (and the regularity of the maps) given in the definition of a lamination is independent of $i$. Since by the Arzela-Ascoli theorem such a sequence of coordinate charts is precompact it follows that a subsequence of the $\gamma_{i}$ 's converges (as a sequence of laminations) to a geodesic lamination $\mathcal{L}$. Implicit in this is that a sequence of laminations is said to converge if the corresponding coordinate charts converge and the local transversals converge as closed subsets of $\mathbb{R}$ in the Hausdorff topology.

It follows from this discussion that if $M^{2}$ is closed with a bumpy metric and $\left\{\gamma_{i}\right\}$ is a sequence of simple closed geodesics, then after passing to a subsequence we may assume that $\gamma_{i} \rightarrow \mathcal{L}$, where $\mathcal{L}$ is a geodesic lamination. Suppose that $\mathcal{L}$ has closed ends and let $\left\{\eta_{j}\right\}$ be the finitely many closed leaves of $\mathcal{L}$ and let $\varepsilon>0$ be sufficiently small so that (2.6) holds. Let $S$ be given by the remarks surrounding Corollary 2.7. Note that in this case, where $\gamma_{i} \rightarrow \mathcal{L}$, the support of $\mathcal{L}$ is connected and each $\eta_{j}$ is a nonisolated leaf, hence strictly stable. If in addition $M^{2}$ does not have a noncompact simple geodesic with a (nontrivial) bounded Jacobi field, then by Proposition 3.9 we can let $\left\{\ell_{k}\right\}_{k=1, \ldots, m+n}$ be the finitely many noncompact leaves of $\mathcal{L}$ ordered so that the first $m$ are the stable leaves. It follows that for $i$ sufficiently large each $\gamma_{i}$ can be decomposed into pieces that spiral very tightly around one of the $\eta_{j}$ 's and pieces that are very close and almost parallel to one of the $\left(\ell_{k} \backslash S\right)$ 's. Note that although there is no a priori bound for how many pieces that circle one of the $\eta_{j}$ 's or are almost parallel to one of the $\left(\ell_{k} \backslash S\right)$ 's for $k \leq m$ and a given $i$, only one piece can be very close to an unstable leaf $\ell_{k}$. Had this last claim not been the case then we would get a contradiction by writing one of the two (disjoint) pieces as a graph over the other and arguing as in Corollary 3.7 to get a positive Jacobi field.

Proposition 3.10. Let $M^{2}$ be a closed surface with a bumpy Kupka-Smale metric. If $\gamma_{i}$ is a collection of simple closed geodesics and every limit of $\left\{\gamma_{i}\right\}$ is a lamination with closed ends, then there is a uniform bound for the Morse indices of $\left\{\gamma_{i}\right\}$.

We say that a bumpy metric on $M^{2}$ is a bumpy Kupka-Smale metric (or bumpy KS-metric) if for each simple stable (noncompact) geodesic with closed ends in $M$, any bounded (normal) Jacobi field vanishes identically. Note that if $M^{2}$ is a closed surface with a bumpy metric, then by the results above the metric is KS if and only if for each simple stable (noncompact) geodesic with closed ends, $J_{-} \neq J_{+}$. 
The proof of Proposition 3.10 will be by contradiction. We will assume that $\gamma_{i}$ is a sequence of simple closed geodesics in a fixed metric and show that the Morse index of these is uniformly bounded. A limit of such a sequence is a geodesic lamination. The relative Poincaré map of one of the $\gamma_{i}$ 's (which will tell us how many zeroes a Jacobi field can have) near the limit lamination will be pieced together from pieces taken from the leaves of the foliation. Thus we will consider first the relative Poincaré map along such a leaf $\gamma$. The next three lemmas examine the three cases: closed leaves $\eta_{j}$, noncompact but stable leaves $\ell_{k}(k \leq m)$ and noncompact unstable leaves $\ell_{k}(k>m)$. We need to watch the image of a fixed vector under the relative Poincaré map to see how many times it can cross the $y$-axis. Recall that such crossings are always transverse and clockwise. In order to prevent the corresponding Jacobi field from having more than one zero we will try to trap it in the right half plane after it crosses the positive $y$-axis.

First let $\eta$ be simple closed and strictly stable. Then using the fact that no Jacobi field has 2 zeroes, it is not difficult to see that the Jacobi fields $J_{ \pm}$have no zeroes; thus the vectors $X_{ \pm}(t)=\left(J_{ \pm}(t), J_{ \pm}^{\prime}(t)\right) \in \mathbb{R}^{2}$ never lie along the $y$-axis. If (according to our convention above) $J_{ \pm}(t)>0$ for all $t$, then $X_{-}$and $X_{+}$both lie to the right of the $y$-axis, and $X_{-}$lies between the positive $y$-axis and $X_{+} \cdot P_{t, s} X_{ \pm}(s)=X_{ \pm}(t)$ and $P_{t, s}$ preserves the four quadrants defined by $\pm X_{ \pm}$. $P_{t+s_{\eta}, t}$ has eigenvectors $X_{-}(t)$, $X_{+}(t)$ with eigenvalues $1 / \lambda, \lambda(0<\lambda<1)$ (the eigenvalues are independent of $\left.t\right)$ thus the directions of the vectors $X_{ \pm}(t)$ are periodic in $t . P_{t+s_{\eta}, t}$ fixes these directions and pushes vectors in the four "quadrants" away from the $X_{+}$-direction and toward the $X_{-}$-direction. Thus the region between the positive $y$-axis and the vector $X_{+}$is a "trap" from which the future orbit of a vector under $P$ cannot escape. Let $X_{0}(t)$ be a (unit) vector in $\mathbb{R}^{2}$ halfway between $X_{ \pm}(t)$.

Lemma 3.11. Let $\eta$ be a strictly stable simple closed geodesic. Then there exist $\varepsilon, H>0$, so that any geodesic, which is the normal graph over $\left.\eta\right|_{[a, b]}$ with $b-a>H$, of a function with norm $<\varepsilon$, has the following property: If $J$ is the Jacobi field with $\left(J(a), J^{\prime}(a)\right)=X_{0}(a)$, then $J$ has no zero in $[a, b]$, and $\left(J(b), J^{\prime}(b)\right)$ lies above $X_{0}(b)$ (in the right half-plane). (Here both the geodesic and its Jacobi field have been reparameterized as graphs.)

Proof. This follows from the fact that by (2.1) the curvature $K$ (as a function of arclength), and thus the relative Poincaré map $P_{t+s_{\eta}, t}$ for the nearby geodesic will be close to that of $\eta$, and the fact that the latter has eigenvalues $\lambda, 1 / \lambda$. This hyperbolicity means that we do not need a bound for $H$.

Lemma 3.11 also follows from Lemma 2.1.

If $\mathcal{L}$ is a geodesic lamination with closed ends on $M^{2}$ with a bumpy metric, then we can find an $\varepsilon$ and an $H$ which will work for each closed leaf. We will assume this in the next two lemmas. 
Now let $\ell$ be noncompact and stable, with no (nontrivial) bounded Jacobi field. Assume $\ell_{-}$and $\ell_{+}$are strictly stable. The vectors $X_{ \pm}(t)=\left(J_{ \pm}(t), J_{ \pm}^{\prime}(t)\right)$ never cross the $y$-axis, and, as above, $X_{-}$lies between the positive $y$-axis and $X_{+} . P_{t, s}$ preserves $\pm X_{ \pm}$and the four quadrants. Let $P_{+}(t)$ be the (nonrelative; ordinary) Poincaré map for $\ell_{+}$, with eigenvectors $V_{ \pm}^{+}(t)$. For $T$ large there is a map $\phi:[T, \infty) \rightarrow \mathbb{R}$ so that $\ell_{+}(\phi(t))$ is a (correctly parameterized) normal graph over $\ell(t)$ on $[T, \infty)$. For fixed $s$,

$$
\lim _{t \rightarrow \infty}\left\|P_{t+s, t}(\ell)-P_{\phi(t)+s, \phi(t)}\left(\ell_{+}\right)\right\|=0 .
$$

Thus in particular when $s=s_{\ell_{+}}$, the period of $\ell_{+}, P_{t+s, t}(\ell)$ will have eigenvectors close to $V_{ \pm}$and eigenvalues close to those of $P_{+}(\phi(t))$. Given $\varepsilon>0, \mu>0$, for $t$ sufficiently large the image under $P_{t, s}(\ell)$ of any vector not within an angle $\mu$ of $\pm X_{+}(s)$ (in $\mathbb{R}^{2}$ "at the time $s$ ") will lie at an angle $<\varepsilon$ of $V_{-}(\phi(t+s)$ ). From this it follows (though different arguments are needed for the two cases) that

$$
\lim _{t \rightarrow \infty}\left\|\frac{X_{ \pm}(t)}{\left\|X_{ \pm}(t)\right\|}-\frac{V_{ \pm}(\phi(t))}{\left\|V_{ \pm}(\phi(t))\right\|}\right\|=0
$$

(As $t$ gets large, $V_{-}$will "soak up" all vectors except $V_{+}$, including $X_{-} . X_{+}$does not get soaked up, and must therefore equal $V_{+}$.) Thus as $\ell$ spirals toward $\ell_{+}$, the basis $\left(X_{-}, X_{+}\right)$approaches the basis $\left(V_{-}, V_{+}\right)$, so that the two relative Poincaré maps can be glued together. Similarly, as $t \rightarrow-\infty$, the basis $\left(X_{-}(t), X_{+}(t)\right)$ approaches a basis $\left(U_{-}(\psi(t)), U_{+}(\psi(t))\right)$ of eigenvectors of $P_{-}(\psi(t))$ for an appropriate reparameterization $\psi$.

Lemma 3.12. For each such (parameterized) geodesic $\ell$, there is an interval $[a, b]$ and $a \delta>0$ so that, for each geodesic $\gamma$ which is the normal graph over $\left.\ell\right|_{[a, b]}$ of a function with norm $<\delta$, the following hold:

1) $\left.\gamma\right|_{[a-H, a]}$ and $\left.\gamma\right|_{[b, b+H]}$ are normal graphs of functions with norm $<\varepsilon$ over closed leaves as in Lemma 3.11.

2) If $J$ is the Jacobifield with $\left(J(a), J^{\prime}(a)\right)=\left(U_{0}(\psi(a))\right.$ (a unit vector midway between $U_{+}(\psi(a))$ and $\left.U_{-}(\psi(a))\right)$, then $J$ has no zero in $[a, b]$, and $\left(J(b), J^{\prime}(b)\right)$ lies above $V_{0}(\phi(b))$ (in the right half-plane). (Here both $\gamma$ and its Jacobi field have been reparameterized as graphs.)

Proof. This is similar to the previous lemma.

Lemma 3.13. Let $\ell$ be a noncompact, unstable leaf. Assume that $\ell_{ \pm}$are strictly stable simple closed geodesics. There is an interval $[a, b], a \delta>0$, and $N \in \mathbb{Z}$ so that, for each geodesic $\gamma$ which is the normal graph over $\left.\ell\right|_{[a, b]}$ of a function with norm $<\delta$,

1) $\left.\gamma\right|_{[a-H, a]}$ and $\left.\gamma\right|_{[b, b+H]}$ are normal graphs of functions with norm $<\varepsilon$ over closed leaves as in Lemma 3.11. 
2) If $J$ is a Jacobi field along $\left.\gamma\right|_{[a, b]}$, then $J$ has at most $N$ zeroes.

We can find $a, b$ and $\delta$ which work simultaneously for all the noncompact leaves.

Proof of Proposition 3.10. Suppose now that there were no bound for the Morse index of all simple closed geodesics on $M$; it follows easily that there exists a sequence $\left\{\gamma_{i}\right\}$ of simple closed geodesics with index $\rightarrow \infty$ and so that $\gamma_{i} \rightarrow \mathscr{L}$, where $\mathscr{L}$ is a geodesic lamination. Let $\left\{\eta_{j}\right\},\left\{\ell_{k}\right\}_{k=1, \ldots, m+n}$ be the leaves of $\mathcal{L}$ as above. Let $H, \varepsilon, \delta, a, b$ be as above. If $i$ is sufficiently large, $\gamma_{i}$ will consist of a union of pieces, each of which lies within $\varepsilon$ of a closed leaf $\eta_{j}$ for a time $>H$, or which (after reparameterization) is a normal graph with norm $<\delta$ over some $\ell_{k} \mid[a, b]$. We can assume that only one piece is a normal graph over $\ell_{k}$ if $k>m$ (by convention $\ell_{k}$ is unstable for $k>m$ ). Let $J$ be a Jacobi field along $\gamma=\gamma_{i}$. We claim that $J$ can have at most $n(N+1)$ zeroes. By Lemmas 3.11, 3.12, if $\left.\gamma_{i}\right|_{[c, d]}$ is a union of pieces as above, but only using the closed leaves $\eta_{j}$ and stable noncompact leaves, then $\left.J\right|_{[c, d]}$ can have at most one zero. Once the vector $\left(J(t), J^{\prime}(t)\right)$ crosses the (say) positive $y$-axis, it will be trapped in the right half-plane, preventing $J$ from having another zero. The claim follows using Lemma 3.13, and thus the proposition.

\section{Genericity of bumpy Kupka-Smale metrics}

We begin with some general comments on the Kupka-Smale hypothesis.

A vector field on manifold is Kupka-Smale (see [PW]) if (1) all closed orbits are hyperbolic, that is, their Poincaré maps do not have any eigenvalue of modulus 1 , and (2) stable and unstable manifolds of closed orbits intersect transversely. Note that without hyperbolicity, in general there are no stable and unstable manifolds, so (2) does not make sense without (1). The Kupka-Smale Theorem states that Kupka-Smale vector fields are generic among $C^{r}$-vector fields, for $r \geq 1$. Our first (dynamical version) definition of KS metric has in mind the Kupka-Smale condition on the vector field generating the geodesic flow on the unit tangent bundle of $M$. There are two differences: First, we are interested only in simple geodesics. Second, we only insist upon hyperbolicity for closed geodesics whose Poincaré maps have real eigenvalues. Note that this includes all stable closed geodesics and all closed ends of simple geodesics, so the manifolds in (2) are indeed manifolds. Since a Poincaré map coming from the geodesic flow on a surface always has determinant 1 , if the eigenvalues are not real there is no hope of pushing them off the unit circle by a small change in metric. Thus in the sense of dynamics of simple geodesics our KS-metric condition is the most one could ask of a generic metric. A careful reader can check that this condition alone is enough to prove Theorems 1.1 and 1.2.

The variational version of the KS-metric condition is also interesting. Condition (1) is a weak version of the bumpy metric condition. It says that, to first order, $\gamma$ 
does not lie locally in a foliation of $M$ by simple closed geodesics. Condition (2) is an analog of the bumpy condition for noncompact geodesics, and appears to be independent of the bumpy metric condition.

In this section we will show that on $M^{2}$ bumpy KS-metrics are generic. Here is the idea: A simple noncompact geodesic $\gamma$ is an intersection point of two circles of geodesics spiraling toward $\gamma_{-}$and $\gamma_{+}$. The intersection will be transverse if the two circles have different tangent vectors at $\gamma$, i.e., if $J_{-} \neq J_{+}$. We will show how to deform the metric so as to make the two circles intersect transversely.

Fix a metric $g$ on $M$ and suppose that $\gamma_{1}, \gamma_{2} \subset M$ are strictly stable simple closed geodesics (where $\gamma_{1}=\gamma_{2}$ is allowed). Let $\Gamma_{-}$be the noncompact (unit speed) geodesics $\gamma$ with $\gamma_{-}=\gamma_{1}$, and $\Gamma_{+}$those with $\gamma_{+}=\gamma_{2}$. If $\gamma \in \Gamma_{-}$, then we let $F_{-}:(-\varepsilon, \varepsilon) \times \mathbb{R} \rightarrow M$ be a (nontrivial) geodesic variation of $\gamma$ so that $F_{-}(s, \cdot) \in \Gamma_{-}, F_{-}(0, \cdot)=\gamma$, and $\left.g\left(\frac{\partial F_{-}}{\partial s}, \frac{\partial F_{-}}{\partial t}\right)\right|_{s=0}=0$. Likewise if $\gamma \in \Gamma_{+}$, then we let $F_{+}$be a (nontrivial) geodesic variation of $\gamma$ consisting of geodesics asymptotic to $\gamma_{2}$. (Note that by Section 3 these variations are essentially unique; we can also assume that $\frac{\partial}{\partial s} F_{ \pm}(0, \cdot)=J_{ \pm}(\gamma)$ and $\left.\frac{\partial^{2}}{\partial s \partial t} F_{ \pm}(0, \cdot)=J_{ \pm}^{\prime}(\gamma)\right)$. We say that $\Gamma_{-}$and $\Gamma_{+}$intersect transversally at $\gamma$ if the two curves representing $\Gamma_{-}$and $\Gamma_{+}$in a local section $\Sigma$ at $\gamma$ are transverse, i.e., if given a curve $v:(-\varepsilon, \varepsilon) \rightarrow M$ with $v(0)=\gamma\left(t_{0}\right)$ for some $t_{0}$ and $v^{\prime}(0)$ transverse to $\gamma^{\prime}(0)$, the curves $\frac{\partial F_{-}}{\partial t} \mid v\left(=X_{-} \mid v\right)$ and $\frac{\partial F_{+}}{\partial t} \mid v\left(=X_{+} \mid v\right)$ in $\Pi^{-1}(v) \subset T_{1} M$ are (well defined and) transverse at 0 . (Note that $\frac{\partial F_{-}}{\partial t}(0,0)=\frac{\partial F_{+}}{\partial t}(0,0)=\gamma^{\prime}\left(t_{0}\right)$.) Now a tangent vector to the curve $\frac{\partial F_{-}}{\partial t} \mid v$

at $s=0$ is given by $\left(v_{*}\left(\frac{d}{d \tau}\right) F_{-}\left(0, t_{0}\right), v_{*}\left(\frac{d}{d \tau}\right) \frac{\partial F_{-}}{\partial t}\left(0, t_{0}\right)\right)$, which is proportional to $\left(J_{-}\left(t_{0}\right) \boldsymbol{n}+\alpha \frac{\partial}{\partial t}, J_{-}^{\prime}\left(t_{0}\right) \boldsymbol{n}\right)$, where $\alpha$ is the slope of the tangent vector to $v$ in the $(s, t)$ coordinates, and similarly for $F_{+}, J_{+}$. Thus transversality means that the vectors $\left(J_{-}\left(t_{0}\right), J_{-}^{\prime}\left(t_{0}\right)\right)$ and $\left(J_{+}\left(t_{0}\right), J_{+}^{\prime}\left(t_{0}\right)\right)$ are not parallel. Since the Jacobi fields are determined uniquely by their and their derivatives values at $t=t_{0}$, by Section 3 transversality of $\Gamma_{-}$and $\Gamma_{+}$at $\gamma$ is equivalent to the fact that $\gamma$ has no bounded nontrivial Jacobi field. To prove that the set of bumpy KS-metrics on $M^{2}$ is residual it suffices therefore (by Lemma 2.6) to show that a residual set of metrics on $M^{2}$ consists of bumpy metrics with the property that, for each pair of strictly stable simple closed geodesics $\gamma_{1}$ and $\gamma_{2}, \Gamma_{-}$and $\Gamma_{+}$intersect transversally.

The rest of this section is devoted to the proof of:

Theorem 4.1. On a closed surface the set of bumpy KS-metrics contains a residual set.

Proof of Theorems 1.1 and 1.2. Theorems 1.1, 1.2 follow by combining Theorem 4.1 with Propositions 3.9, 3.10, respectively.

In Lemmas 4.2, 4.4 below we let $M^{2}$ be a closed surface with metric $g$ and $c:\left[-\varepsilon, r_{0}+\varepsilon\right] \rightarrow M$ be a simple $C^{m+2}$ curve parameterized by arclength. Let $\boldsymbol{n}_{c}$ 
be the unit normal (so $\boldsymbol{n}_{c} \in C^{m+1}$ ) and let $\Phi:\left[-\varepsilon, s_{0}+\varepsilon\right] \times[0, \varepsilon] \rightarrow M$ be given by $\Phi(s, t)=\exp _{c(s)}\left(t \boldsymbol{n}_{c(s)}\right)$ so that $\Phi^{-1}$ are geodesic normal coordinates in a neighborhood of $c$. In these the metric is of the form $f^{2}(s, t) d s^{2}+d t^{2}$ where $f \in C^{m}$. (Note that in any metric of this form, the curves $s=$ constant are minimal and thus geodesic.)

We will give a simple direct argument to show that the set of strictly bumpy metrics on $M$ is dense. The following deformation lemma will be needed to show that on a surface for a dense set of metrics certain geodesic variations intersect transversally. The lemma allows us to alter the geodesic flow in a controlled way by altering the metric.

Lemma 4.2. Let $M^{2}, c, \Phi$ be as above. Let $c_{w}:\left[-\varepsilon, s_{0}+\varepsilon\right] \rightarrow M$ be the curve $c_{w}(s)=\Phi(s, \varepsilon-w s)$. There exists a 1-parameter family of $C^{m}$ metrics $g_{w}$ $(w \in(-\delta, \delta))$ such that $g_{0}=g$, each $g_{w}=g$ on $M \backslash \Phi\left(\left[-\varepsilon, s_{0}-\varepsilon\right] \times[0,3 \varepsilon / 4]\right)$, $\Phi_{w}(s, \varepsilon)=c_{w}(s)$ for $s \in\left[0, s_{0}\right]$, and $\frac{\partial}{\partial t} \Phi_{w}(s, \varepsilon)$ points perpendicular to the curve $c_{w}(s)$. (Here $\Phi_{w}^{-1}$ are geodesic normal coordinates in a neighborhood of $c$ in the metric $g_{w}$.) That is, in the $g_{w}$ metric, the geodesics which enter the box $\Phi$ perpendicular to the curve $c(s)$ exit perpendicular to the curve $c_{w}(s)$.

Proof. Fix $w>0$ sufficiently small. Let $\Psi_{w}^{-1}$ be geodesic normal coordinates in a neighborhood of $c_{w}$ parameterized so that $\Psi_{w}(s, t):\left[-\varepsilon, s_{0}+\varepsilon\right] \times[0, \varepsilon] \rightarrow M$, and $\Psi_{w}(s, \cdot)$ are geodesics moving away from $c$ and ending up on $c_{w}$ orthogonal to $c_{w}$. In particular $\Psi_{w}(s, \varepsilon)=c_{w}(s)$. In these coordinates the metric can be written as $f_{w}^{2}(s, t) d s^{2}+d t^{2}$. Let $\eta:[0, \varepsilon) \rightarrow[0,1]$ be a smooth cutoff function with $\eta \mid[0, \varepsilon / 2]=1$. Then $\Phi_{\eta, w}^{-1}(s, t)=\left(\eta(t) \Phi^{-1}(s, t)+(1-\eta(t)) \Psi_{w}^{-1}\right)(s, t)$ is a diffeomorphism and gives therefore local coordinates $(s, t)$. In these define a metric by $\hat{g}_{w}(s, t)=\left(\eta(t) f(s, t)+(1-\eta(t)) f_{w}(s, t)\right)^{2} d s^{2}+d t^{2}$. Finally, let $\phi \in C_{0}^{\infty}\left(-\varepsilon, s_{0}+\varepsilon\right)$ with $0 \leq \phi \leq 1, \phi \mid\left[0, s_{0}\right]=1$, and set $g_{w}(s, t)=$ $\phi(s) \hat{g}_{w}(s, t)+(1-\phi(s)) g(s, t)$. It is easy to see that this gives a 1-parameter family with the desired properties.

We will use this deformation to make the family $\Gamma_{-}$(locally) transverse to $\Gamma_{+}$. The metric will be deformed in a rectangle $\Phi$ to change the family $\Gamma_{-}$as it moves through the rectangle, (roughly speaking) before it meets the family $\Gamma_{+}$at the top of the rectangle. To see the effect of this deformation of the metric on the image of the family of geodesics $\Gamma_{-}$in the local section $\Sigma$ given by the (fixed) curve $c_{0}$ at the top of the rectangle, we will need to know the angle at which these geodesics cross the curve $c_{0}$. The image of the family in $\Sigma$ is (in appropriate coordinates) the graph of the crossing angle as a function of arclength along $c_{0}$. Lemma 4.4 below begins by showing that the deformation of the metric given in Lemma 4.2 moves this graph (and thus the curve which is the image of the family $\Gamma_{-}$in $\Sigma$ ) off itself. The following version of Sard's theorem says that if we can move a curve in the plane off itself, 
we can make it transverse to a second curve. (Here we say that the intersection of the image of $h$ and the graph of $f$ is transverse if $h^{\prime}(t)=\left(\frac{d x}{d t}, \frac{d y}{d t}\right)$ is transverse to $\left(1, f^{\prime}(x)\right)$ for every $(x, y)$ and $t$ with $h(t)=(x, y)$.)

Lemma 4.3. Let $f_{w}(s)$ be $C^{1}$ functions, where $s \in[0,1]$ and $w \in(-\delta, \delta)$. Let $h: \mathbb{R} \rightarrow \mathbb{R}^{2}$ be $C^{1}$. If $\left|\frac{\partial f_{w}}{\partial w}\right|_{w=0}$ is nonvanishing, then there exists a sequence $w_{i} \rightarrow 0$ such that the curves $\left(s, f_{w_{i}}(s)\right)$ are transverse to $h$.

Proof. By a $C^{1}$ change of coordinates, we can assume the functions $f_{w}(s)$ are constant. In these coordinates transversality of $h$ and $f_{w}$ is equivalent to $f_{w}$ being a regular value of $\pi_{2} \circ h$, where $\pi_{2}$ is projection onto the second factor in $\mathbb{R}^{2}$. By Sard's theorem the claim easily follows.

Lemma 4.4. Let $M^{2}, c$, $\Phi$ be as above with $m=0$. If $h:[0,1] \rightarrow T_{1} M \mid \Phi(\cdot, \varepsilon)$ is a $C^{1}$ curve, then there exists a sequence of metrics $g_{i} \rightarrow g$ with $g_{i}=g$ on $M \backslash \Phi\left(\left[-\varepsilon, s_{0}+\varepsilon\right] \times[0,3 \varepsilon / 4]\right)$ and such that in any $g_{i}, \frac{\partial \Phi_{i}}{\partial t}$ intersects $h$ transversally along $\Phi(\cdot, \varepsilon) \mid\left[0, s_{0}\right]$. Here $\Phi_{i}^{-1}$ are geodesic normal coordinates in a neighborhood of $c$ in the metric $g_{i}$.

Proof. Assume first that $c$ is actually $C^{\infty}$. As above let $\Phi^{-1}=(s, t)$ be geodesic normal coordinates in a neighborhood of $c$ so that we can in particular think of $t$ as a function on this neighborhood. Moreover, in this neighborhood the metric is $f^{2}(s, t) d s^{2}+d t^{2}$. Let $g_{w}, \Phi_{w}$ be given by Lemma 4.2 and set $h_{w}=\frac{\partial \Phi_{s}}{\partial t} \mid \Phi(\cdot, \varepsilon)$. It follows from Lemma 4.2 that $\left|\frac{\partial}{\partial w}\right| w=0, h_{w} \mid$ is nonvanishing for $s \in\left[0, s_{0}\right]$. Namely, it is easy to see that $h_{w}$ is $C^{1}$ so we need only check that the derivative is nonvanishing. To see this let $\gamma_{w}:\left[\theta_{0}, \theta_{w}\right] \rightarrow M$ be a (unit speed) geodesic (in the metric $g$ ) with $\gamma_{w}\left(\theta_{0}\right) \in\{\Phi(s, \varepsilon-w s)\}, \gamma_{w}^{\prime}\left(\theta_{0}\right)$ orthogonal to $\{\Phi(s, \varepsilon-w s)\}$, and $\gamma_{w}\left(\theta_{w}\right) \in$ $\{\Phi(s, \varepsilon)\}$. Set $u(\theta)=\left(t \circ \gamma_{w}\right)^{\prime}(\theta)=g\left(\gamma_{w}^{\prime}(\theta), \nabla t\right)$, then $u^{\prime}(\theta)=\operatorname{Hess}_{t}\left(\gamma_{w}^{\prime}, \gamma_{w}^{\prime}\right)=$ $\left(1-u^{2}(\theta)\right) \frac{f^{\prime}}{f}$. Hence, $\left|(\log [(1+u) /(1-u)])^{\prime}\right| \leq C$, where $C=C(g)$ is a constant. In particular,

$$
\left|\frac{\left(1+u\left(\theta_{w}\right)\right)}{\left(1-u\left(\theta_{w}\right)\right)} \frac{\left(1-u\left(\theta_{0}\right)\right)}{\left(1+u\left(\theta_{0}\right)\right)}\right| \leq \exp \left(C\left|\theta_{w}-\theta_{0}\right|\right) .
$$

Moreover, it is easy to see that for some $\alpha=\alpha(g)$ and some $\beta=\beta(g)>0$

$$
\left|\theta_{w}-\theta_{0}\right| \leq \alpha w \text { and }\left|u\left(\theta_{0}\right)\right| \geq \beta w .
$$

Combining (4.1) with (4.2) we conclude that for some $\beta^{\prime}=\beta^{\prime}(g)>0$

$$
\left|u\left(\theta_{w}\right)\right| \geq \beta^{\prime} w .
$$

As $g_{w}=g$ on $M \backslash \Phi\left(\left[-\varepsilon, s_{0}+\varepsilon\right] \times[0,3 \varepsilon / 4]\right)$ it follows easily from (4.3) that $\left|\frac{\partial}{\partial w}\right|_{w=0} h_{w} \mid$ is nonvanishing for $s \in\left[0, s_{0}\right]$ and the lemma follows from Lemma 4.3. 
In the general case where $c$ is only $C^{2}$ let $c_{j}$ be a sequence of $C^{\infty}$ curves in $M$ with $\left|c-c_{j}\right|_{C^{2}} \rightarrow 0$. It follows easily from the continuity of the quantities involved that for $j$ sufficiently large (but fixed) if $g_{w, j}$ and $\Phi_{w, j}$ are given by Lemma 4.2 with respect to $c_{j}$ and $h_{w, j}=\frac{\partial \Phi_{w, j}}{\partial r} \mid \Phi(\cdot, \varepsilon)$, then $\left|\frac{\partial}{\partial w}\right|_{w=0} h_{w, j} \mid$ is nonvanishing. The lemma now easily follows from Lemma 4.3.

Let again $M^{2}$ be a closed surface with a metric $g$ and suppose that $\gamma_{1}, \gamma_{2}$ are strictly stable simple closed geodesics (where $\gamma_{1}=\gamma_{2}$ is allowed). Let $\Gamma_{-,+}^{k}$ be the $\gamma \in \Gamma_{-} \cap \Gamma_{+}$with $\gamma \backslash T_{\delta}\left(\gamma_{1} \cup \gamma_{2}\right)$ of length $\leq k$.

The next result will follow by applying Lemma 4.4 a finite number of times.

Lemma 4.5. Let $M^{2}, g, \gamma_{1}$, and $\gamma_{2}$ be as above. Given $k>0$, there exists $g_{n} \rightarrow g$ with $g_{n}=g$ in a neighborhood of $\gamma_{1} \cup \gamma_{2}$ and such that for each $g_{n}, \Gamma_{-}\left(g_{n}\right)$ and $\Gamma_{+}\left(g_{n}\right)$ intersect transversally at $\Gamma_{-,+}^{k}\left(g_{n}\right)$.

Proof. Fix $\delta$ small but positive. Let $\sigma_{1}: \mathbb{S}^{1} \rightarrow M$ be a simple closed curve in $T_{\delta / 2}\left(\gamma_{1}\right)$ meeting each geodesic in $\Gamma_{-}$exactly once (Corollary 3.4). Parameterize $\Gamma_{-}$as a map $F_{-}: \mathbb{S}^{1} \times \mathbb{R} \rightarrow T_{1} M$ with $F_{-}(s, 0)=\sigma_{1}(s)$. Similarly let $\sigma_{2}: \mathbb{S}^{1} \rightarrow T_{\delta / 2}\left(\gamma_{2}\right)$ meet each geodesic in $\Gamma_{+}$exactly once, and let

$$
F_{+}: \mathbb{S}^{1} \times \mathbb{R} \rightarrow T_{1} M \quad \text { with } F_{+}(s, 0)=\sigma_{2}(s) .
$$

(Of course we want $F_{-} \rightarrow \gamma_{1}$ as $t \rightarrow-\infty$ and $F_{+} \rightarrow \gamma_{2}$ as $t \rightarrow \infty$.) Given $\varepsilon$ sufficiently small, and $x \in T_{\delta / 2}\left(\gamma_{1}\right)$, we can find (Corollary 3.6) a unit speed curve $c:[-2 \varepsilon, 2 \varepsilon] \rightarrow T_{\delta}\left(\gamma_{1}\right)$ with $c(0)=x$, and which is everywhere perpendicular to the vector field $X_{-}$. Let $\Phi:[-2 \varepsilon, 2 \varepsilon] \times[-\varepsilon, 0] \rightarrow M$ be geodesic normal coordinates, with $\Phi(s, 0)=c(s)$ and $\Phi(s, \cdot) \in \Gamma_{-}$(when extended) for each $s$. Given $k$, and $\gamma \in \Gamma_{-}$, there exist $\varepsilon, \mu>0$ (depending only upon $k$, not on $\gamma$ ) and $t_{0} \in[-1,0]$ (depending upon $k$ and $\gamma$ ) so that this box $\Phi$ for the point $x=\gamma\left(t_{0}\right)$ has the following properties:

a) Geodesics entering the bottom $(t=-\varepsilon)$ of the box vertically (tangent to $s=$ constant) have never been in the box before.

b) Geodesics leaving the top $(t=0)$ of the box at an angle less than $\mu$ of the vertical travel at least a distance $5 k$ before returning within $\varepsilon$ of the box.

Let $\hat{c}:[-2 \varepsilon, 2 \varepsilon] \rightarrow \Pi^{-1}(c)$ be the lift of the vector field $X_{-}: \hat{c}=\left.X_{-}\right|_{c}$. Note that by a) above, $\hat{c}$ is the intersection of the local section $\Sigma=\Pi^{-1}(c) \subset T_{1} M$ given by the transversal $c$, with the image of $\left.F_{-}\right|_{(-\infty, 0]}$. Let $D$ be the disk in $\Sigma$ consisting of all vectors in $\Pi^{-1}(c)$ making an angle less than $\mu$ with $X_{-}$. Consider the image in $T_{1} M$ of $\left.F_{+}\right|_{[-k, \infty)}$. This "tube" will intersect $D$ transversely if $\mu<\pi / 2$. The tube will thus intersect $D$ in a union $C_{k}$ of $C^{1}$ curves whose total length is finite. An arbitrarily 
small alteration $g_{w}$ of the metric in the box will not change the intersection $C_{k}$ of $D$ with $\left.F_{+}\right|_{[-k, \infty)}$ by b) above, but will, by Lemma 4.4, make $\left.\hat{c}\right|_{[-\varepsilon, \varepsilon]}$ transverse to $C_{k}$.

Given $k$ we can find a finite number of such boxes $\Phi$ with each geodesic in $\Gamma_{-}$ intersecting some $\left.c\right|_{[-\varepsilon, \varepsilon]}$. Thus we will be done if we can make each $\left.\hat{c}\right|_{-\varepsilon, \varepsilon]}$ transverse to $C_{k}$. Now when we alter the metric in the second box it may change the image in the section $D$ at the top of the first box, of the forward tube $\left.F_{+}\right|_{[-k, \infty)}$. However a sufficiently small change of metric in the second box will not destroy transversality for the first box due to the fact that, as a consequence of Corollary 3.4, transversality is an open property.

Since transversality of the sets $\Gamma_{-}(g)$ and $\Gamma_{+}(g)$ along $\Gamma_{-,+}^{k}(g)$ for any fixed $k>0$ is an open property in $g$ (this follows easily from Corollary 3.6) we get:

Corollary 4.6. Let $M^{2}, g, \gamma_{1}$, and $\gamma_{2}$ be as above. If $\Gamma_{-}(g)$ and $\Gamma_{+}(g)$ intersect transversally at $\Gamma_{-,+}^{k}(g)$, then there exists an open neighborhood $U$ of $g$ such that for each $\bar{g} \in U, \Gamma_{-}(\bar{g})$ and $\Gamma_{+}(\bar{g})$ intersect transversally at $\Gamma_{-,+}^{k}(\bar{g})$.

Proof. Suppose not; it follows easily that there exists $g_{i} \rightarrow g, \gamma_{1, i} \rightarrow \gamma_{1}, \gamma_{2, i} \rightarrow \gamma_{2}$, and $\gamma_{i} \in \Gamma_{-,+}^{k}\left(g_{i}\right)$ with $\left(\frac{\partial F_{-, i}}{\partial s}, \frac{\partial^{2} F_{-, i}}{\partial s \partial t}\right)=\left(\frac{\partial F_{+, i}}{\partial s}, \frac{\partial^{2} F_{, i}}{\partial s \partial t}\right)$ at $\gamma$. Clearly $\gamma_{i} \rightarrow \gamma \in$ $\Gamma_{-,+}^{k}(g)$ and by Corollary 3.6 it follows easily that $\left(\frac{\partial F_{-}}{\partial s}, \frac{\partial^{2} F_{-}}{\partial s \partial t}\right)=\left(\frac{\partial F_{+}}{\partial s}, \frac{\partial^{2} F_{+}}{\partial s \partial t}\right)$ at $\gamma$ which is the desired contradiction.

Proof of Theorem 4.1. Fix integers $L, k>0$ and let $g_{L, k}$ be the set of metrics $g$ on $M^{2}$ with the following two properties:

a) All closed geodesics with length $<L$ are nondegenerate critical points.

b) If $\gamma_{1}, \gamma_{2}$ are simple closed strictly stable geodesics with length $<L$, then $\Gamma_{-}(g)$ and $\Gamma_{+}(g)$ intersect transversally along $\Gamma_{-,+}^{k}(g)$.

Combining the fact that the set of bumpy metrics is dense (see [Ab], [An]) with Lemmas 2.6, 4.5, and Corollary 4.6 we get that $g_{L, k}$ is open and dense, hence $\bigcap_{L, k>0} g_{L, k}$ is residual.

\section{Geodesic laminations without closed ends; Theorem 1.3}

A train-track is a one-complex $T$ embedded in a surface satisfying conditions of (1) smoothness, (2) nondegeneracy, and (3) geometry. The definition is quite involved, and probably familiar to many readers. Rather than attempt an abridged, incorrect version, we refer the reader to [HaPe] (see p. 4 there) for this and other definitions.

Let $F$ be a disk with four holes removed, see Figure $3 \mathrm{a}$, and let $N$ be the topological double of $F$ so $N$ is a closed orientable surface of genus 4. Equip $N$ with a metric with negative curvature so that the boundary of $F \subset N$ consists of geodesics. 


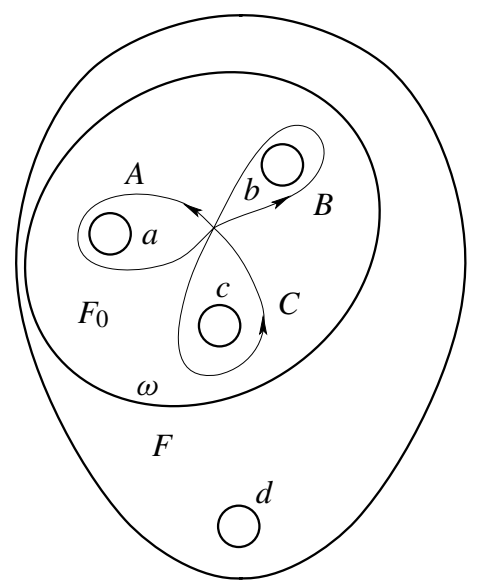

Figure 3a. The disk-minus-4-holes $F$, the disk-minus-3-holes $F_{0}$, and generators $A, B, C$ for the fundamental group of $F_{0}$.

On $F$ pick three of the holes; there is a unique closed geodesic $\omega$ on $F$ enclosing these three holes but not the fourth. Let $F_{0} \subset F$ be the disk bounded by $\omega$, with the three holes removed. The fundamental group of $F_{0}$ is a free group on three generators $A, B, C$, one for each hole. Figure $3 b$ shows a transversely recurrent train-track $T$

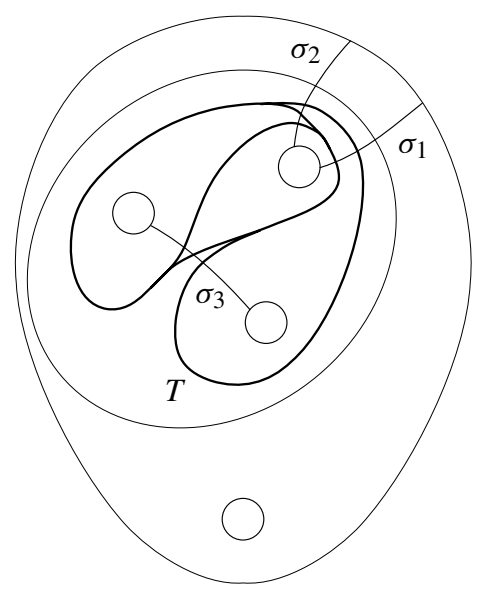

Figure 3b. The traintrack $T$ and the closed curves $\sigma_{i}$ on the double $N$ of $F$.

on $N$. (That $T$ satisfies the "geometry" condition for a train-track is clear from the characterization on the top of p. 6 in [HaPe]: The complement of $T$ in $N$ is connected, and clearly has no component that is an embedded nullgon, monogon, bigon, annulus, 
or punctured annulus. Transverse recurrence can be seen by looking at the closed curves on $N$ that are the doubles $\sigma_{i}$ of the curves in Figure $3 \mathrm{~b}$. The reader will easily see how to find curves as the $\sigma_{i}$ 's so that each branch of $T$ meets at least one of the $\sigma_{i}$, and for each $i$, the complement in $N$ of $T \cup \sigma_{i}$ is either connected, or has two components, one of which is an embedded trigon. Since neither component is an embedded bigon, each $\sigma_{i}$ hits $T$ efficiently ([HaPe], p. 19).)

In Figure $3 \mathrm{c}$ we see a simple curve $\rho$ on $F$ that for $t>0$ is carried on this traintrack. The bounded homotopy class (that is, we only consider homotopies that move points a bounded distance in the universal cover) of the forward end of the curve $\rho$

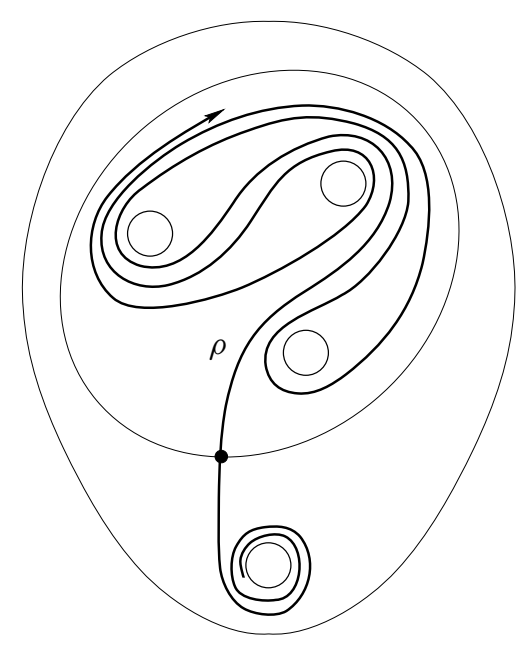

Figure 3c. The curve $\rho$.

determines a (semi-)infinite word $B A^{-1} B^{-1} C B A B^{-1} A \ldots$ in the generators $A, B$ and $C$. Theorem 1.3 will follow from two lemmas:

Lemma 5.1. An uncountable number of different words in A, B, C come from different completions of the forward end of $\rho$ as a simple curve carried on the train-track $T$.

Lemma 5.2. Each such completion of $\rho$ is bounded-homotopic in $F$ to a simple geodesic.

Proof of Lemma 5.1. In Figure 3c each of the holes $a, b, c$ lies in a snake-like cavity bounded by a segment of $\rho$. Two of these cavities open out where $\rho$ meets $\omega$; the third ends at the forward end of $\rho$. The retraction of $\rho$ onto $T$ retracts the head of the snake to the boundary of a monogon (the monogon actually lies in the disk, before the holes have been removed to form $F$ ) whose interior contains no other branches 
of $T$, and identifies the two sides of each snake's body, so a simple curve carried by $T$ that enters one of these cavities has exactly two ways to continue up to homotopy: Clockwise or counterclockwise. These properties will persist as we complete the curve $\rho$.

At present the forward end of $\rho$ lies at the top of the picture. It will proceed around to the bottom, and enter the cavity containing hole $a$. The reader can easily verify that when one snake eats another as in Figure 4a the possibilities are as follows: Suppose

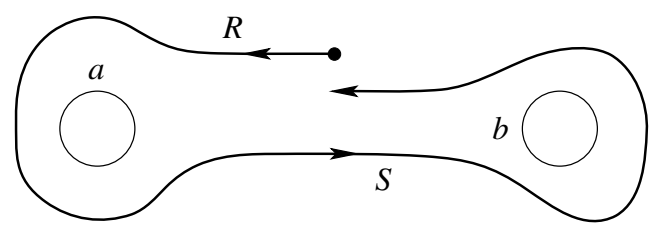

Figure 4a. One snake about to eat another.

the left snake has the word $R$ in the letters $A, B, C$, and the right snake the word $S$. ( $R$ is conjugate to $A$, and $S$ to $B$.)

(1) The word $(R S)^{k} R S R^{-1}(R S)^{-k}, k \geq 0$.

(2) The word $(R S)^{k} R(R S)^{-k}, k \geq 1$.

(3) The infinite word $R S R S R S R S R \ldots$

In the first two cases, the forward end emerges as in Figure 4b. It will then proceed to enter the third cavity, and the above possibilities repeat.

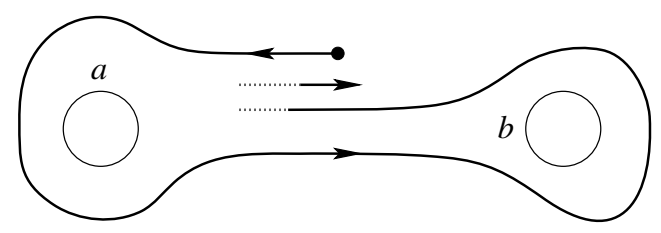

Figure $4 \mathrm{~b}$. The tail of the second snake emerges.

The important things for us are: There are no "wrong turns", that is the curve can always be completed as a simple curve carried by $T$; and the curve never runs out of possibilities, that is there is always another choice of words in the future. It follows that an uncountable number of different words results.

Proof of Lemma 5.2. Let $\tilde{N}$ be the universal cover of $N$ and $\tilde{N}(\infty)$ the sphere (circle) at infinity of $\tilde{N}$; see for instance [Eb]. Let $\tilde{\rho}$ be a lift of $\rho$ to $\tilde{N}$, with $p$ the point lying over the intersection of $\rho$ with $\omega$. Let $\tilde{\omega}$ be the lift of $\omega$ through $p$, and $\tilde{F}$ be the lift 
of $F$ through $p$. By proposition 1.5.1 of [HaPe] (that proposition is stated in case of constant negative curvature but the argument extends) $\tilde{\rho}$ has two (unique, and distinct) limit points $q, r$ on $\tilde{N}(\infty)$. Let $\tilde{\ell}$ be the geodesic on $\tilde{N}$ with these two limit points. If $\tilde{\tau}$ is a geodesic on $\tilde{N}$ with neither $q$ nor $r$ as a limit point, then the topological intersection numbers $\#(\tilde{\rho}, \tilde{\tau})$ and $\#(\tilde{\ell}, \tilde{\tau})$ are well defined and equal. Because $\tilde{N}$ has negative curvature, the number of points of intersection of two geodesics in $\tilde{N}$ is 0 or 1 . It follows that $\ell$ lies on $F$, and on $F_{0}$ after crossing $\omega$. Moreover, if $\tilde{\sigma}$ is a geodesic segment, with boundary on $\partial \tilde{F}$, then $\#(\tilde{\rho}, \tilde{\sigma})=\#(\tilde{\ell}, \tilde{\sigma})$. The reader can easily verify that for each branch of $T$ there is a curve $\tau$ on $F_{0}$, with boundary on $\partial F_{0}$, that intersects $T$ exactly once. By making $\rho$ stick close to $T$, we can assume that $\tau$ and $\rho$ intersect once each time (and only when) $\rho$ follows the given branch of $T$ (i.e., when the retraction of $\rho$ onto $T$ includes the branch). Each lift of $\tau$ to $\tilde{F}_{0}$ that meets $\tilde{\rho}$, say at a point $p_{i}$, is homotopic to a geodesic $\tilde{\sigma}_{i}$ with boundary on $\partial \tilde{F}_{0}$, and $\tilde{\sigma}_{i}$ will intersect $\tilde{\ell}$ exactly once, say at $q_{i}$. We can now define a homotopy from $\tilde{\rho}$ to $\tilde{\ell}$ that starts by taking each point $p_{i}$ to $q_{i}$; then "pull tight" to straighten out the curve in between the points $q_{i}$. Thus $\tilde{\rho}$ is homotopic to $\tilde{\ell}$ inside $\tilde{F}$, and $\rho$ is homotopic to $\ell$ inside $F$, by a homotopy that does not move any point very far.

It remains only to see that $\ell$ is simple. Since $\rho$ is simple, $\tilde{\rho}$ does not intersect any other lift of $\rho$. The same intersection number argument as before shows that $\tilde{\ell}$ does not intersect any other lift of $\ell$, which implies that $\ell$ is simple.

Proof of Theorem 1.3. First we will produce an open set of metrics on $M$ having geodesic laminations without closed ends. Fix a metric on $N$ with constant curvature -1 ( $F$ is one-half of $N$ ) and let $U \subset N$ be an open neighborhood of $F$. On the given surface $M$ we can complete the metric on $U$ to a metric on $M$. Any nearby metric on $M$ will contain a (unique) metric surface $F \subset U$ of negative curvature and geodesic boundary that also has a completion to a metric of negative curvature on $N$.

The statement now follows from the lemmas as follows: If $\ell$ is the geodesic in $F$ isotopic to $\rho$, and $\ell$ has closed ends, by Lemma 2.4 the word determined by $\rho$ will eventually repeat. But uncountable many of the words determined by simple completions $\rho$ will be nonrepeating. For such $\rho$ the closure of $\ell$ will be a lamination of $F$ with nonclosed ends.

Next we find a (smaller) open set of metrics, each having a geodesic lamination without closed ends that is the limit of a sequence of simple closed geodesics.

Let $F_{1}$ be a topological disk-minus-6-holes. As before we assume a metric of negative curvature on $F_{1}$ with geodesic boundary, that extends to a metric of negative curvature on the topological double $N_{1}$ of $F_{1}$. Let $\omega$ be a geodesic enclosing holes $a$, $b, c$ but not holes $d, e, f$. Put the train-track $T$ inside $\omega$ as before, and a similar traintrack $S$ around the holes $e, f, g$. As before we can find a simple curve $\rho$ on $F_{1}$ that crosses $\omega$ once, and whose bounded homotopy class determines a (doubly) infinite word starting in $d, e, f$ and ending in $a, b, c$, and not repeating at either end. We will 
next describe a sequence of simple closed curves $\rho_{k}$ on $F_{1}$, which "approximate" $\rho$. The curve $\rho_{k}$ starts at a point on $\omega$, and follows the path of $\rho$ in and out of $k-1$ cavities. In the $k$ 'th cavity, the curve $\rho_{k}$ will turn around counterclockwise, and then stick close to the (just laid out) strand of $\rho_{k}$ on its left, until it retraces its steps back to the intersection with $\omega$. The other half of the path of $\rho_{k}$ does the same thing on the other side, then closes up. There will be a simple closed geodesic $\gamma_{k}$ in the free homotopy class of $\rho_{k}$. Take lifts $\tilde{\rho}_{k}$ through a fixed point $p$ lying above $\omega$, and corresponding lifts $\tilde{\gamma}_{k}$. The curve $\rho$ will cross (in order) a sequence $\tilde{\sigma}_{j}$ of lifts of (a finite number of) geodesic segments $\sigma_{j}$ on $F_{1}$, with boundary on $\partial F_{1}$, that keep track of which choice was made at each branch. The same will be true of the geodesics $\gamma_{k}$, as long as $\rho_{k}$ follows $\rho$. Now fix $J$. By the Arzela-Ascoli theorem, for each $J$ any limit lamination of the sequence $\gamma_{k}$ will contain a geodesic $\ell_{J}$ with a lift that has the same intersection with the $\sigma_{j},\|j\| \leq J$, as $\rho$. Finally, the closure of the set of geodesics $\ell_{J}$ in the unit tangent bundle contains a geodesic $\ell$ with a lift that intersects all of (and only) the segments $\tilde{\sigma_{j}}$ intersecting $\rho$, in order. This geodesic does not have closed ends, since its "word" is the same as that of $\rho$ (note that their lifts are bounded-homotopic), and hence nonrepeating. By construction any limit lamination of the sequence $\gamma_{k}$ must contain the geodesic $\ell$.

Fix the metric of constant curvature -1 on $N_{1}$, and let $U$ be an open neighborhood of $F_{1}$ in $N_{1}$. For a given surface $M$, any metric extending the metric surface $U$ will possess a neighborhood consisting of metrics, each of which has a geodesic lamination without closed ends that is the limit of a sequence of simple closed geodesics.

\section{References}

[Ab] R. Abraham, Bumpy metrics. In Global Analysis, Proc. Sympos. Pure Math. XIV, Amer. Math. Soc., Providence, R.I., 1968, 1-3. Zbl 0215.23301 MR 0271994

[An] D. V. Anosov, Generic properties of closed geodesics. Izv. Akad. Nauk SSSR Ser. Mat. 46 (1982), 675-709, 896 (in Russian). Zbl 0512.58014 MR 0670163

[Bi] G. D. Birkhoff, Dynamical Systems. Amer. Math. Soc. Colloq. Publ. 9, Amer. Math. Soc., New York 1927. JFM 53.0732.01

[CD] T. H. Colding and C. De Lellis, Singular limit laminations, Morse index, and positive scalar curvature. Topology 44 (2005), 25-45. Zbl 02137337 MR 2103999

[CH1] T. H. Colding and N. Hingston, Metrics without Morse index bounds. Duke Math. J. 119 (2003), 345-365. Zbl 1059.53037 MR 1997949

[CH2] T. H. Colding and N. Hingston, Morse index bounds for simple geodesics on surfaces. In preparation.

[CM1] T. H. Colding and W. P. Minicozzi II, Examples of embedded minimal tori without area bounds. Internat. Math. Res. Notices 1999 (1999), 1097-1100. Zbl 0980.53084 MR 1728019 
[CM2] T. H. Colding and W. P. Minicozzi II, Embedded minimal surfaces without area bounds in 3-manifolds. In Geometry and topology (Aarhus 1998), Contemp. Math. 258, Amer. Math. Soc., Providence, RI, 2000, 107-120. Zbl 0999.53006 MR 1778099

[Eb] P. Eberlein, Geometry of nonpositively curved manifolds. Chicago Lectures in Mathematics, University of Chicago Press, Chicago, Il, 1996. Zbl 0883.53003 MR 1441541

[HaPe] J. L. Harer and R. C. Penner, Combinatorics of train tracks. Ann. of Math. Stud. 125, Princeton University Press, Princeton, NJ, 1992. Zbl 0765.57001 MR 1144770

[HaNoRu] J. Hass, P. Norbury, and J. H. Rubinstein, Minimal spheres of arbitrarily high Morse index. Comm. Anal. Geom. 11 (2003), 425-439. MR 2015753

[HiPu] M. W. Hirsch and C. Pugh, Stable manifolds and hyperbolic sets. In Global Analysis, Proc. Sympos. Pure Math XIV, Amer. Math. Soc., Providence, R.I., 1970, 133-165. Zbl 0215.53001 MR 0271991

[Kl] W. P. A. Klingenberg, Riemannian geometry. Second edition, de Gruyter Stud. Math. 1, Walter de Gruyter and Co., Berlin 1995. Zbl 0911.53022 MR 1330918

[KIT] W. P. A. Klingenberg and F. Takens, Generic properties of geodesic flows. Math. Ann. 197 (1972) 323-334. Zbl 0225.58006 MR 0307282

[MS] D. McDuff and D. Salamon, Introduction to Symplectic Topology. Oxford Mathematical Monographs, The Clarendon Press, Oxford University Press, New York 1995. Zbl 0844.58029 MR 1373431

[PW] J. Palis and M. Welington, Geometric Theory of Dynamical Systems. SpringerVerlag, New York, Berlin 1982. Zbl 0491.58001 MR 0669541

[PiRu] J. Pitts and J. H. Rubinstein, Applications of minimax to minimal surfaces and the topology of three-manifolds. In Geometry and partial differential equations (Canberra 1986), Proc. Centre Math. Anal. Austral. Nat. Univ. 12, Austral. Nat. Univ., Canberra 1987, 137-170. Zbl 0639.49030 MR 0924434

[Sp] M. Spivak, A comprehensive introduction to differential geometry. Second edition, Vol. 4, Chapter 8, Publish or Perish, Inc., Wilmington, Del., 1979. Zbl 0439.53004 MR 0532833

Received February 20, 2003; revised September 21, 2005

Tobias H. Colding, MIT, Department of Mathematics, 77 Massachusetts Avenue, Cambridge, MA 02139-4307, U.S.A.

E-mail: colding@cims.nyu.edu

Nancy Hingston, Department of Mathematics, The College of New Jersey, Ewing, NJ 08628, U.S.A.

E-mail: hingston@TCNJ.EDU 